COMMUNICATIONS IN

ANALYSIS AND GEOMETRY

Volume 14, Number 4, 795-845, 2006

\title{
Higher canonical asymptotics of Kähler-Einstein metrics on quasi-projective manifolds
}

\author{
DAMIN WU
}

\begin{abstract}
We derive a canonical asymptotic expansion up to infinite order of the Kähler-Einstein metric on a quasi-projective manifold, which can be compactified by adding a divisor with simple normal crossings. Characterized by the log filtration of the Cheng-Yau Hölder ring, the asymptotics are obtained by constructing an initial Kähler metric, deriving certain iteration formula and applying the isomorphism theorems of the Monge-Ampère operators. This work is parallel to the asymptotics of Fefferman, Lee and Melrose on pseudoconvex domains in $\mathbb{C}^{n}$.
\end{abstract}

\section{Introduction}

On a complex manifold $M$ of dimension $n$, a volume form $\Psi$ is a smooth positive $(n, n)$ form. In a local coordinate neighborhood $U_{\alpha}$ with holomorphic coordinates $z_{\alpha}=\left(z_{\alpha}^{1}, \ldots, z_{\alpha}^{n}\right)$,

$$
\Psi=\xi_{\alpha} \prod_{j=1}^{n}\left(\frac{\sqrt{-1}}{2 \pi} d z_{\alpha}^{j} \wedge d \bar{z}_{\alpha}^{j}\right)
$$

where $\xi_{\alpha}$ is a positive $C^{\infty}$ function. Then the Ricci form Ric $\Psi$ associated to $\Psi$ is the real $(1,1)$ form given locally by

$$
\operatorname{Ric} \Psi=d d^{c} \log \xi_{\alpha},
$$

where $d^{c}:=\sqrt{-1} / 4 \pi(\bar{\partial}-\partial)$. It follows that Ric $\Psi$ is globally defined on $M$ and that $\operatorname{Ric} \Psi=c_{1}\left(K_{M}\right)$, where $K_{M}$ is the canonical bundle.

We define the generalized Fefferman operator $J$ by

$$
J(\Psi)=\frac{(\operatorname{Ric} \Psi)^{n}}{\Psi}
$$


where $\Psi$ is a volume form. Let $\Psi_{\varphi}=e^{\varphi} \Psi$. Then we have the following iteration formula

$$
J\left(\Psi_{\varphi}\right)=M_{\operatorname{Ric} \Psi}(\varphi) J(\Psi)
$$

where

$$
M_{\operatorname{Ric} \Psi}(\varphi)=\frac{\left(\operatorname{Ric} \Psi+d d^{c} \varphi\right)^{n}}{(\operatorname{Ric} \Psi)^{n}} e^{-\varphi},
$$

which is the Monge-Ampère operator associated to Ric $\Psi$. It follows from the definition that $J\left(\Psi_{\varphi}\right)=1$ if and only if $M_{\operatorname{Ric} \Psi}(\varphi)=1 / J(\Psi)$.

Let $M$ be a complex manifold. A divisor $D$ on $M$ is said to have normal crossings if locally $D$ is given by an equation

$$
z_{1} \cdots z_{k}=0
$$

where $\left(z_{1}, \ldots, z_{n}\right)$ are local holomorphic coordinates on $M$. Moreover, if each irreducible component of $D$ is smooth, then we shall say that $D$ has simple normal crossings.

Let $\bar{M}$ be a compact complex manifold and $D$ be a divisor in $\bar{M}$ with simple normal crossings. We impose the positivity condition

$$
K_{\bar{M}}+[D]>0 \text {. }
$$

Then, a theorem of Carlson-Griffiths [5] assures that there exists a volume form $\Omega$ on $M \equiv \bar{M} \backslash D$ such that $\operatorname{Ric} \Omega>0$ on $M$, and $(M, \operatorname{Ric} \Omega$ ) is a complete Kähler manifold with negative Ricci curvature.

This theorem provides an initial metric $\omega \equiv \operatorname{Ric} \Omega$, which can be deformed to the canonical Kähler-Einstein metric on $M$. In fact, this was first addressed by Yau [40] and later on by Cheng and Yau [9], Kobayashi [22], Tsuji [35], Tian and Yau [34] and Bando [2]. It follows that $M$ possesses a unique complete Kähler-Einstein metric of constant negative Ricci curvature -1 .

However, the results on the existence of Kähler-Einstein metrics on $M$ are not enough when people want to know the singular description, in the form of asymptotic expansion, of the canonical metric near the divisor $D$. Actually, this geometric information would help us to develop broader and deeper applications of the theory of quasi-projective manifolds to differential and algebraic geometry. This was suggested by Yau [42, p. 377].

In this paper, we derive an asymptotic expansion of the Kähler-Einstein metrics near a simple normal crossing divisor $D$. In order to characterize 
the asymptotics, we introduce the weighted Cheng-Yau Hölder rings and the associated filtrations based on the bounded geometry. We first develop the analysis of the Monge-Ampère operators on these weighted spaces. We derive certain isomorphism theorems for the Monge-Ampère operators and their linearizations. These results imply that the Monge-Ampère operators and the Laplacians preserve the log filtration of the Cheng-Yau Hölder ring up to infinite weight.

Secondly, we construct certain initial volume form which can be used to approximate the canonical volume form in a nice way. This construction depends on the existence of a canonical metric on the smooth subvariety. Thirdly, by using the initial volume form and the iteration formula (1.1) we can derive a formal asymptotic expansion of the Kähler-Einstein volume form; then, by the isomorphism theorems, we prove that the formal asymptotics is indeed the real asymptotics. Furthermore, we prove that the obtained asymptotics is canonical in the sense that it is independent of the extensions of the canonical metric on the subvariety.

The plan of this paper is to start from deriving the asymptotics in a special case to make it easier for the reader to comprehend what is going on before treating the more involved simple normal crossing case. The whole machinery derived in the smooth case is generalized in a less trivial way to overcome the difficulties arisen from the higher codimensional situations. Also, the proof of canonicity requires some further development of the analysis on the bounded geometry.

In Section 2, we establish the basic setting, the bounded geometry and the weighted Cheng-Yau Hölder spaces, which are essential for our geometric analysis. The notions of local quasi-coordinate map, bounded geometry and the Cheng-Yau Hölder spaces were introduced in [8, 9, 34]. For completeness, we include them in the first subsection. Then in Section 2.2, we introduce the weighted Cheng-Yau Hölder rings and the associated filtrations, which will be used to characterize the asymptotics. Furthermore, we define the differential forms over the weighted rings, which are convenient for the later analysis.

In Section 3, we derive the asymptotics in the case of complement of a smooth divisor $D$. In section 3.1, we establish the isomorphism theorems for both the Monge-Ampère operator $M_{\omega}$ and its linearization. To do this, we first linearize $M_{\omega}$ as certain negative Laplacian $\Delta_{u}$ by fixing the solution $u$. Then, we construct a family of linear elliptic operators which can be viewed as certain conjugacies of $\Delta_{u}-1$. By the Schauder theory and Yau's generalized maximum principle, we derive a theorem of Fredholm alternative for such elliptic operators, which, in turn, implies the isomorphism theorems. 
In section 3.2, the solution $u$ of the Monge-Ampère equation

$$
M_{\omega}(u)=J(\Omega)^{-1}
$$

can be characterized by the weight 1 Cheng-Yau Hölder ring $\mathcal{R}_{1}(M)$. This is obtained from a careful construction of the initial metric, which depends on a canonical metric on the smooth divisor $D$. This characterization result, followed immediately from the isomorphism theorem for $M_{\omega}$, is independent of the extension of the canonical metric. In Section 3.3, a formal asymptotic expansion is derived by the perturbation method based on the iteration formula (1.1). The crucial part is to prove that this formal asymptotics is the real asymptotics, which is achieved by the isomorphism theorems derived in Section 3.1.

In Section 4, the whole machinery used in the previous section is generalized to derive the asymptotics near a divisor with simple normal crossings. More precisely, we obtain the asymptotics of Kähler-Einstein volume form near the complete intersections of the irreducible components of the divisor. In Section 4.1, we establish the isomorphism theorems on the homogeneous weighted Cheng-Yau Hölder spaces with respect to an index subset $I$, which is essential in the proof of asymptotics in the simple normal crossing case. Moreover, our isomorphism theorems are formulated and proved in a much general form, which, we believe, should have interests of their own.

The construction of initial volume forms in Section 4.2 is less trivial than that of the smooth case. In fact, we study the construction from the view-point of "moduli spaces"; namely, we consider $\mathcal{M}_{I}$ and $\mathcal{M}_{I, \mathcal{H}_{I}}$, the families of the initial volume forms which satisfy the adjunction formula, and the formula plus certain compatibility condition, respectively. The latter condition is useful in characterizing the canonicity of the asymptotics. In Theorem 4.8, we present a general way to extend the metric while preserving the positivity of the curvature form on any higher codimensional subvarieties of complete intersection. We believe such a metric extension theorem should also have interests of its own.

Finally, together with the results in the previous sections and the iteration formula, in Section 4.3, we derive the canonical asymptotics near the complete intersection. Namely, given $\Omega \in \mathcal{M}_{I}$, the Kähler-Einstein volume form $\Omega_{K-E}=e^{u} \Omega$ on $M$ has the following canonical asymptotic expansion near the complete intersection $D_{I}$ :

$$
\Omega_{K-E} \sim\left(1+\sum_{\left|r_{I}\right|=1}^{\infty} \frac{\phi_{r_{I}}}{\sigma^{r_{I}}}\right) \Omega,
$$


where $\phi_{r_{I}} \in \mathcal{R}(M)$ for each $r_{I} \in \mathbb{Z}_{+}^{|I|}$, and $\sigma^{r_{I}}$ is the monomial involving the logarithm of norms of defining sections of the irreducible components $D_{i}$ with $i \in I$ (see Section 2.2 for the definitions). The asymptotics is canonical in the the following sense: Let $\Omega \in \mathcal{M}_{I, \mathcal{H}_{I}}$. If (1.4) is derived in terms of another $\Omega^{\prime} \in \mathcal{M}_{I, \mathcal{H}_{I}}$, and coefficients $\left\{\phi_{r_{I}}^{\prime}\right\}_{r_{I} \in \mathbb{Z}_{+}^{|I|}}$, then

$$
\begin{aligned}
u^{\prime}-u & \in \mathcal{R}_{I, \infty}(M) \\
\phi_{r_{I}}^{\prime}-\phi_{r_{I}} & \in \mathcal{R}_{I, \infty}(M), \quad \text { for any } r_{I} \in \mathbb{Z}_{+}^{|I|} .
\end{aligned}
$$

Our work may be viewed as the counterpart of Fefferman [15] and LeeMelrose's asymptotics [24] on pseudoconvex domains in $\mathbb{C}^{n}$. The background of their work refers to Fefferman's papers [14,15, 16,3] and Cheng-Yau [8]. See also Bland [4], Graham [18], Hirachi [21] and the references therein for the further development of the asymptotic geometry of pseudoconvex domains and Cauchy-Riemann manifolds.

In the special case of a smooth divisor, certain initial results and applications were obtained by Schumacher in $[32,33]$, in which he used the idea of using the canonical metric on the divisor to construct an initial volume form on $M$. His main result is equivalent to $u \in \mathcal{R}_{r}(M)$ with $0<r \leq 1$ undetermined, which is, however, less precise. Indeed, the accurate weight, $r=1$, is crucial for deriving the asymptotics, as in Section 3.3. Also, the continuity method does not give information on the higher order terms of the asymptotics.

Our work completely settles the general case of a simple normal crossing divisor. The method was motivated in [24,23]. Indeed, we first obtained a formal asymptotic expansion in terms of the log filtration. The theorem of Fredholm alternative derived in Section 3.1 enables us to prove the isomorphism theorems on Cheng-Yau Hölder spaces up to infinite weight. These, in turn, imply that the formal asymptotics is the real asymptotics, which is furthermore canonical provided the initial volume forms satisfy certain compatibility condition.

The asymptotics near a simple normal crossing divisor can be viewed as a higher dimensional generalization of Nevanlinna's classic result on $\mathbb{P}^{1} \backslash\left\{p_{1}, \ldots, p_{\mu} \mid \mu \geq 3\right\}[29$, p. 249-250], which played a fundamental role in the second main theorem of the Nevanlinna theory. Hence, it is natural to expect that this work could have applications to the modern Nevanlinna theory, which, in turn, has applications to transcendental algebraic geometry (see, for example, $[5,10,20]$ ). 


\section{Bounded geometry}

We first recall the notions of local quasi-coordinate map, bounded geometry and the Cheng-Yau Hölder spaces in Section 2.1. In Section 2.2, we consider the initial volume form and the associated initial metric, which can be deformed to the Kähler-Einstein metric. Furthermore, to characterize the asymptotics, we introduce the weighted Ching-Yau Hölder rings and the associated filtrations, which will be used extensively in the following sections.

\subsection{Quasi-coordinate map and bounded geometry}

Let $X$ be an $n$-dimensional complex manifold. Recall that the notion of quasi-coordinate is given as follows:

Definition 2.1. Let $V \subset \mathbb{C}^{n}$ be an open set. A holomorphic map $\phi: V \rightarrow$ $X^{n}$ is called a quasi-coordinate map if $\operatorname{rank}_{p}(d \phi)=n$ for every $p \in V$. In this case, $(V, \phi)$ is called a local quasi-coordinate chart of $X$.

Next, the bounded geometry is defined below in terms of a system of local quasi-coordinates.

Definition 2.2. Given a complete Kähler manifold $(X, \omega)$, we say that $(X, \omega)$ has bounded geometry of order $m+\mu$, where $m \in \mathbb{Z}_{+}$, and $\mu \in[0,1)$, if there exists a system of local quasi-coordinates $\mathcal{V}=\left\{\left(V_{\eta}, \phi_{\eta}\right)\right\}$ such that

(1) $X=\bigcup_{\eta} \phi_{\eta}\left(V_{\eta}\right)$, and each $x \in X$ is centered at some $V_{\eta}$;

(2) For each $\eta, 1 / 2 \leq$ radius of $V_{\eta} \leq 1$;

(3) There exist constants $C$ and $\mathcal{A}_{m}$ such that, for each $\eta$, if we write

$$
\phi_{\eta}^{*}(\omega) \equiv \frac{\sqrt{-1}}{2 \pi} \sum_{i, j=1}^{n} g_{\eta, i \bar{j}} d v^{i} \wedge d \bar{v}^{j}
$$

then

$$
\begin{gathered}
0<C^{-1}\left(\delta_{i j}\right) \leq\left(g_{\eta, i \bar{j}}\right) \leq C\left(\delta_{i j}\right), \\
\left\|g_{\eta, i \bar{j}}\right\|_{C^{m, \mu}\left(V_{\eta}\right)} \equiv \sup _{|p|+|q| \leq m}\left\|\frac{\partial^{|p|+|q|}}{\partial v^{p} \partial \bar{v}^{q}} g_{\eta, i \bar{j}}\right\|_{C^{\mu}\left(V_{\eta}\right)} \leq \mathcal{A}_{m} .
\end{gathered}
$$


A fundamental example is the unit punch disk $\Delta^{*}=\{0<|z|<1\}$ in $\mathbb{C}$ together with the Poincaré metric

$$
\omega_{\Delta^{*}}=\frac{\sqrt{-1}}{2 \pi} \frac{d z \wedge d \bar{z}}{|z|^{2}\left(\log |z|^{2}\right)^{2}} .
$$

Let $V_{\eta} \equiv \Delta_{3 / 4}:=\{v \in \mathbb{C} ;|v|<3 / 4\}$ and

$$
\phi_{\eta}(v)=e^{(1+\eta) /(1-\eta) \cdot(v+1) /(v-1)}, \quad \text { for each } \eta \in(0,1) .
$$

Then $\cup_{0<\eta<1} \phi_{\eta}\left(V_{\eta}\right)=\Delta^{*}$, and the family $\left\{\left(V_{\eta}, \phi_{\eta}\right)\right\}$ forms a system of local quasi-coordinates of $\Delta^{*}$ such that $\left(\Delta^{*}, \omega_{\Delta^{*}}\right)$ has bounded geometry of order infinity. Here, the essential point is the invariance of Poincaré metrics under the map $\phi_{\eta}$.

Based on the bounded geometry, the Cheng-Yau's Hölder spaces are defined as follows:

Definition 2.3. Fix a quasi-coordinate system $\mathcal{V}=\left\{\left(V_{\eta}, \phi_{\eta}\right)\right\}$ with (2.2), (2.2), and (2.2) stated in Definition 2.2. For $k \in \mathbb{Z}_{+}, \alpha \in[0,1)$, define the norm $\|\cdot\|_{k, \alpha}$ on $C^{\infty}(X)$ by

$$
\|u\|_{k, \alpha}=\sup _{V_{\eta} \in \mathcal{V}}\left\{\left\|\phi_{\eta}^{*}(u)\right\|_{C^{k, \alpha}\left(V_{\eta}\right)}\right\} .
$$

Define

$$
\begin{gathered}
C^{k, \alpha}(X)=\text { the completion of }\left\{u \in C^{\infty}(X) ;\|u\|_{k, \alpha}<+\infty\right\} \\
\text { with respect to }\|\cdot\|_{k, \alpha} .
\end{gathered}
$$

Define the Cheng-Yau Hölder ring by

$$
\mathcal{R}(X)=\bigcap_{k \geq 0,0<\alpha<1} C^{k, \alpha}(X)
$$

\subsection{Weighted Cheng-Yau Hölder rings and the associated filtrations}

Let $\bar{M}$ be a compact complex manifold and $D=\sum_{i=1}^{p} D_{i}$ be a simple normal divisor, where the irreducible components $D_{i}$ are smooth and intersect transversely. We let $M=\bar{M} \backslash D$. Assume that $K_{\bar{M}}+[D]>0$. Let 
$s_{i} \in H^{0}\left(\bar{M}, \mathcal{O}\left(\left[D_{i}\right]\right)\right)$ be the holomorphic section defining $D_{i}$. Then, by the theorem of Carlson and Griffiths, there exists a $C^{\infty}$ volume form $V$ on $\bar{M}$ and a sufficiently small metric on each $\left[D_{i}\right]$ such that

$$
\Omega \equiv \frac{V}{\prod_{i=1}^{p}\left|s_{i}\right|^{2}\left(\log \left|s_{i}\right|^{2}\right)^{2}}
$$

is a volume on $M$ satisfying the following properties:

(i) Ric $\Omega>0$ on $M$, and $(M, \operatorname{Ric} \Omega)$ is a complete Kähler manifold with finite volume;

(ii) there is a positive constant $C$ such that

$$
C^{-1}<J(\Omega)<C \quad \text { on } \bar{M} \text {. }
$$

Such a volume form $\Omega$ is called an initial volume form. Denote

$$
\begin{aligned}
\omega & =\operatorname{Ric} \Omega \\
& =\omega_{K}+2 \sum_{i=1}^{p} \sigma_{i}^{-1} \omega_{c_{i}}+2 \sum_{i=1}^{p} \sigma_{i}^{-2} d \sigma_{i} \wedge d^{c} \sigma_{i},
\end{aligned}
$$

where

$$
\begin{aligned}
\omega_{K} & =\operatorname{Ric}\left(\frac{V}{\prod_{i=1}^{p}\left|s_{i}\right|^{2}}\right), \\
\omega_{c_{i}} & =-d d^{c} \sigma_{i}, \\
\sigma_{i} & =-\log \left|s_{i}\right|^{2}, \quad i=1, \ldots, p .
\end{aligned}
$$

It is well known that $(M, \omega)$ has a system of local quasi-coordinates $\left(V_{\eta}, \phi_{\eta}\right)$ with bounded geometry of order infinity (see, for example, $[9,22,34]$ ). In fact, the local quasi-coordinates are inspired from the fundamental example given in Section 2.1. We only need to be careful near the divisor $D$. Suppose that in a neighborhood $\left(U,\left\{z^{1}, \ldots, z^{n}\right\}\right)$ of $p \in D$ we have $D \cap U=\left\{z^{1} \cdots z^{k}=0\right\}=\left(\Delta^{*}\right)^{k} \times \Delta^{n-k}$, where $\Delta$ is the unit disk in $\mathbb{C}$. Let $V_{\eta} \equiv\left(\Delta_{3 / 4}\right)^{k} \times \Delta^{n-k}$ and $\phi_{\eta}(v)=\left(\phi_{\eta}^{1}(v), \ldots, \phi_{\eta}^{n}(v)\right)$ with

$$
\begin{aligned}
& \phi_{\eta}^{i}(v)=e^{\left(1+\eta^{i}\right) /\left(1-\eta^{i}\right) \cdot\left(v^{i}+1\right) /\left(v^{i}-1\right)}, \quad i=1, \ldots, k, \\
& \phi_{\eta}^{j}(v)=v^{j}, \quad j=k+1, \ldots, n,
\end{aligned}
$$


for each $\eta \in(0,1)^{k}$. Then $U$ is covered by $\cup_{\eta \in(0,1)^{k}} \phi_{\eta}\left(V_{\eta}\right)$. Let $U$ run through all the neighborhoods. We get a system of local quasi-coordinates of $M$ such that $(M, \omega)$ has bounded geometry of order infinity.

Denote by $\mathbb{R}_{+}$and $\mathbb{Z}_{+}$the sets of non-negative real numbers and nonnegative integers, respectively; denote $\mathbb{R}_{+}^{m} \equiv\left(\mathbb{R}_{+}\right)^{m}$ and $\mathbb{Z}_{+}^{m} \equiv\left(\mathbb{Z}_{+}\right)^{m}$ for each $m \in \mathbb{N}$. For $r=\left(r_{1}, \ldots, r_{p}\right) \in \mathbb{R}_{+}^{p}$ with $|r| \equiv r_{1}+\cdots+r_{p}$, denote by

$$
\sigma^{-r}=\sigma_{1}^{-r_{1}} \cdots \sigma_{p}^{-r_{p}} .
$$

Then the weighted Cheng-Yau Hölder spaces $\sigma^{-r} C^{k, \alpha}(M)$ are the Banach spaces defined as usual. For each $l \in \mathbb{N}$, the homogeneous weighted space $\sum_{|r|=l} \sigma^{-r} C^{k, \alpha}(M)$ is defined as a normed linear subspace of $C^{k, \alpha}(M)$. Similarly, let

$$
\mathcal{R}_{r}(M)=\sigma^{-r} \mathcal{R}(M), \quad \text { for any } r \in \mathbb{R}_{+}^{p} .
$$

Moreover, denote by $\mathcal{R}^{1}(M)$ the $\mathcal{R}(M)$-module of differential 1-forms on $M$; namely, for each 1 -form $\varphi \in \mathcal{R}^{1}(M)$, for any $k \in \mathbb{Z}_{+}$, there exist a constant $\mathcal{A}_{\varphi, k}$ such that if, for each local quasi-coordinate $\left(V_{\eta}, \phi_{\eta}\right)$,

$$
\phi_{\eta}^{*}(\varphi)=f_{i} d v^{i}+g_{\bar{j}} d \bar{v}^{j}
$$

then

$$
\left\|f_{i}\right\|_{C^{k, \alpha}\left(V_{\eta}\right)} \leq \mathcal{A}_{\varphi, k}, \quad\left\|g_{\bar{j}}\right\|_{C^{k, \alpha}\left(V_{\eta}\right)} \leq \mathcal{A}_{\varphi, k}, \quad \text { for any } \alpha \in(0,1) .
$$

Note that $\mathcal{R}^{1}(M)$ is a subset of $A^{1}(M)$, the set of smooth 1 -forms on $M$. Let

$$
\mathcal{R}^{m}(M)=\wedge^{k} \mathcal{R}^{1}(M) \text { for any } m \in \mathbb{N} .
$$

Similarly, denote by $\mathcal{R}^{p, q}(M)$ the module of $(p, q)$-forms on $M$ over the ring $\mathcal{R}(M)$. In particular, it follows from the definition that $\omega \in \mathcal{R}^{1,1}(M)$; furthermore, a $(p, p)$-form $\varphi$ on $M$ belongs to $R^{p, p}(M)$ implies that

$$
\Lambda_{\omega}(\varphi) \equiv \frac{\omega^{n-p} \wedge \varphi}{\omega^{n}} \in \mathcal{R}(M) .
$$

We are interested in the asymptotic behavior of the Kähler-Einstein metric near the (non-empty) complete intersections,

$$
D_{I} \equiv D_{k_{1}} \cap \cdots \cap D_{k_{i}}
$$

where the index set

$$
I=\left\{i_{1}, \ldots, i_{k}\right\} \subset\{1, \ldots, p\}
$$


satisfies that

$$
D_{j} \cap D_{I}=\emptyset \quad \text { for any } j \notin I .
$$

It is convenient to denote by $\mathcal{I}$ the collection of all such index subsets. By the definition of simple normal crossing, each $D_{I}, I \in \mathcal{I}$, is a smooth subvariety of codimension $|I|, 1 \leq|I| \leq n$, but is not necessary connected. Write

$$
D_{I}=\sum_{\nu} D_{I}^{\nu}
$$

where each $D_{I}^{\nu}$ is a connected component. So, more precisely, we are interested in the asymptotics near each connected component $D_{I}^{\nu}$.

To characterize the asymptotics, we introduce the weighted Cheng-Yau Hölder rings associated to $D_{I}, I=\left\{i_{1}, \ldots, i_{k}\right\} \in \mathcal{I}$, as below:

$$
\begin{aligned}
\mathcal{R}_{I, t}(M) & =\tau_{I}^{t} \mathcal{R}(M), \quad \text { for any } t \in \mathbb{R}_{+} . \\
\mathcal{R}_{I, \infty}(M) & =\bigcap_{t \geq 0} \mathcal{R}_{I, t}(M),
\end{aligned}
$$

where the weight function

$$
\tau_{I} \equiv\left(\sum_{i \in I} \sigma_{i}^{-2}\right)^{1 / 2} .
$$

They are all ideals of $\mathcal{R}(M)$. Moreover, it follows from the definition that, for each $l \in \mathbb{N}$,

$$
\begin{aligned}
\mathcal{R}_{I, l}(M) & =\left(\sum_{i \in I} \sigma_{i}^{-1}\right)^{l} \mathcal{R}(M) \\
& =\sum_{\left|r_{I}\right|=l} \sigma^{-r_{I}} \mathcal{R}(M)
\end{aligned}
$$

where

$$
\sigma^{-r_{I}}=\prod_{i \in I} \sigma_{i}^{-r_{i}}, \quad r_{I}=\left(r_{i_{1}}, \ldots, r_{i_{k}}\right) \in \mathbb{Z}_{+}^{|I|}
$$

Furthermore, we can also define the differential forms over the weighted Cheng-Yau Hölder rings as follows:

$$
\begin{array}{rlrl}
\mathcal{R}_{I, k}^{m}(M) & \equiv \tau_{I}^{k} \mathcal{R}^{m}(M), & & \mathcal{R}_{I, k}^{p, q}(M) \equiv \tau_{I}^{k} \mathcal{R}^{p, q}(M), \\
\mathcal{R}_{I, \infty}^{m}(M) & \equiv \bigcap_{k \geq 0} \mathcal{R}_{I, k}^{m}(M), & \mathcal{R}_{I, \infty}^{p, q}(M) \equiv \bigcap_{k \geq 0} \mathcal{R}_{I, k}^{p, q}(M) .
\end{array}
$$


for any $m, k, p, q \in \mathbb{Z}_{+}$. By the definitions and (2.3), we have

$$
\Lambda_{\omega}\left(\mathcal{R}_{I, k}^{p, p}(M)\right) \subset \mathcal{R}_{I, k}(M),
$$

for any $m, k, p, q \in \mathbb{Z}_{+}$. The similar results also hold for the Cheng-Yau Hölder rings with infinite weight.

Any sequence of non-decreasing numbers $\left\{t_{j}\right\}_{j \in \mathbb{N}} \subset \mathbb{R}_{+}$gives rise to a filtration of $\mathcal{R}(M)$ :

$$
\mathcal{R}_{I, t_{1}}(M) \supset \mathcal{R}_{I, t_{2}}(M) \supset \mathcal{R}_{I, t_{3}}(M) \supset \cdots .
$$

Now we consider the $\log$-filtration $\left\{\mathcal{R}_{I, k}(M)\right\}_{k \in \mathbb{Z}_{+}}$of $\mathcal{R}(M)$. Define the graded Cheng-Yau Hölder ring $\mathcal{R}_{I}^{G}(M)$ associated to the log filtration by

$$
\begin{aligned}
& \mathcal{R}_{I}^{G}(M)=\{u \in \mathcal{R}(M) \mid \text { there exist a multiple sequence } \\
& \left\{\psi_{r} \in \mathcal{R}(M) \mid r \in \mathbb{Z}_{+}^{p}, r_{i}=0 \text { if } i \notin I\right\}, \text { where } \\
& \text { not all } \psi_{r} \text { are zero, such that } u \sim \sum_{|r|=0}^{\infty} \psi_{r} \sigma^{-r} \text {, i.e., } \\
& \text { for each } \left.N \in \mathbb{N}, u-\sum_{|r|=0}^{N} \psi_{r} \sigma^{-r} \in \mathcal{R}_{I, N+1}(M) \text {. }\right\} \text {. }
\end{aligned}
$$

Let

$$
\mathcal{R}_{I, k}^{G}(M)=\mathcal{R}_{I}^{G} \bigcap \mathcal{R}_{I, k}(M), \quad \text { for each } k \in \mathbb{N} .
$$

In the special case that $D$ is smooth, we have

$$
\mathcal{R}_{I, t}(M)=\mathcal{R}_{t}(M)=\sigma^{-t} \mathcal{R}(M), \quad \text { for all } t \in \mathbb{R}_{+},
$$

where

$$
\sigma=-\log |s|^{2}
$$

in which $s \in H^{0}(\bar{M}, \mathcal{O}[D])$ defines $D$. In this case, $\mathcal{R}_{k}(M)_{k \in \mathbb{Z}_{+}}$gives rise to the log filtration of $\mathcal{R}(M)$. Similarly, we have the graded Cheng-Yau Hölder rings $\mathcal{R}_{k}^{G}(M)$ for each $k \in \mathbb{Z}_{+}$. We will first derive the asymptotics in this special case, which is the content of the next section.

\section{Smooth divisor case}

The approach of deriving the asymptotics near a smooth divisor consists of three parts: the first part is to establish the isomorphism theorems for both 
the Monge-Ampère operator and its linearizations. The second part is to construct certain initial volume form which can approximate the KählerEinstein volume form in a nice way. Finally, by the initial volume form and the iteration formula, we derive the asymptotics, which is proved to be the real asymptotics by the isomorphism theorems.

\subsection{Isomorphism theorems}

Let $\bar{M}$ be a compact complex manifold and $D$ be a smooth divisor. Denote $M=\bar{M} \backslash D$. Suppose $K_{\bar{M}}+[D]>0$. Let $s \in H^{0}(\bar{M}, \mathcal{O}([D]))$ to be a holomorphic section defining $D$. Then, there exists a $C^{\infty}$ volume form $V$ on $\bar{M}$ such that

$$
\Omega=\frac{V}{|s|^{2} \log ^{2}|s|^{2}}
$$

is an initial volume form on $M$. (See (2.2) and (2.2) in Section 2.) Denote $\omega=\operatorname{Ric}(\Omega)$ and $\sigma=-\log |s|^{2}$. Then,

$$
\omega=\omega_{K}+2 \sigma^{-1} \omega_{c}+2 \sigma^{-2} d \sigma \wedge d^{c} \sigma
$$

in which

$$
\begin{aligned}
\omega_{K} & =\operatorname{Ric}\left(\frac{V}{|s|^{2}}\right)>0, \\
-\omega_{c} & =d d^{c} \sigma \in c_{1}([D]), \quad \text { on } M .
\end{aligned}
$$

Let $C^{k, \alpha}(M), k \geq 0$ and $\alpha \in(0,1)$ be the Cheng-Yau Hölder spaces formed by the local quasi-coordinates. Then we have the first isomorphism theorem as follows:

Theorem 3.1. Fix an arbitrary $r \in \mathbb{R}_{+}, k \geq 0$ and $\alpha \in(0,1)$. For each $F \in \sigma^{-r} C^{k, \alpha}(M)$, let $u \in C^{k+2, \alpha}(M)$ be the unique solution of

$$
\begin{aligned}
M_{\omega}(u) & \equiv \frac{\left(\omega+d d^{c} u\right)^{n}}{\omega^{n}} e^{-u}=e^{F}, \\
\frac{1}{C} \omega & \leq \omega+d d^{c} u \leq C \omega, \quad C>0 .
\end{aligned}
$$

Then, $u \in \sigma^{-r} C^{k+2, \alpha}(M)$. 
Proof of Theorem 3.1. (3.2) implies that

$$
u+F=\log \frac{\left(\omega+d d^{c} u\right)^{n}}{\omega^{n}} .
$$

Let $\omega_{t}=\omega+t d d^{c} u$. Then it follows that

$$
\begin{aligned}
u+F & =\int_{0}^{1}\left[\frac{d}{d t} \log \left(\frac{\omega_{t}^{n}}{\omega^{n}}\right)\right] d t \\
& =\int_{0}^{1}\left(\frac{n \omega_{t}^{n-1} \wedge d d^{c} u}{\omega_{t}^{n}}\right) d t
\end{aligned}
$$

i.e., we can view the Monge-Ampère Equation (3.2) as the following "linear" equation:

$$
\left(\Delta_{u}-1\right) u=F
$$

where

$$
\Delta_{u}(v) \equiv \int_{0}^{1}\left(\frac{n \omega_{t}^{n-1} \wedge d d^{c} v}{\omega_{t}^{n}}\right) \mathrm{d} t, \quad \text { for all } v \in C^{2}(M)
$$

Therefore, it suffices to show that

$$
\Delta_{u}-1: \sigma^{-r} C^{k+2, \alpha}(M) \longrightarrow \sigma^{-r} C^{k, \alpha}(M)
$$

is an isomorphism.

Observe that (3.3) implies

$$
\left[\frac{t}{C}+(1-t)\right] \omega \leq \omega_{t} \leq[C t+(1-t)] \omega, \quad \text { for all } t \in[0,1]
$$

which assures that $\Delta_{u}-1$ is uniformly elliptic in each local quasi-coordinate. It follows from Yau's generalized maximum principle and Schauder's theory that

$$
\Delta_{u}-1: C^{k+2, \alpha}(M) \longrightarrow C^{k, \alpha}(M)
$$

is a linear homeomorphism. Now we want to construct a linear operator

$$
L_{u, r}: C^{k+2, \alpha}(M) \longrightarrow C^{k, \alpha}(M),
$$


which is uniformly elliptic in each local quasi-coordinate chart, such that the following diagram commutes:

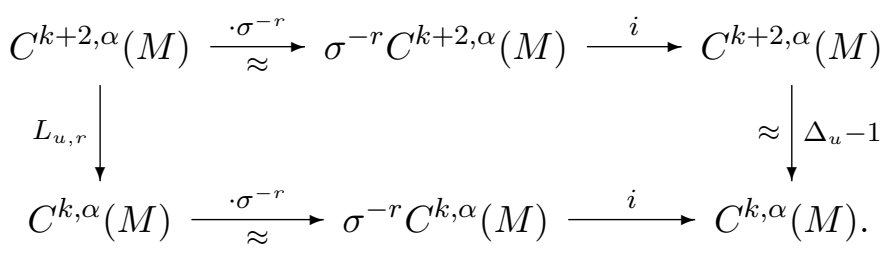

(In this paper, the map $i$ stands for the inclusion unless otherwise indicated.)

Assume that (3.8) is true and that $L_{u, r}$ is an isomorphism; then the proof is finished since (3.8) will give rise to the following commutative diagram:

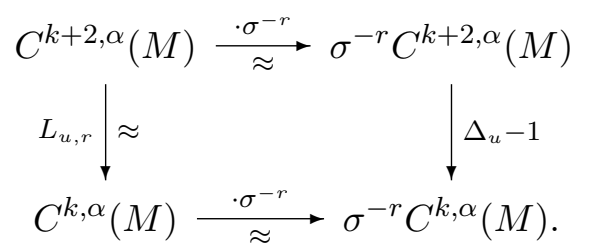

Therefore, it remains to construct an isomorphism $L_{u, r}$ such that (3.8) holds. Now for each $v \in C^{k, \alpha}(M)$, we define

$$
\begin{aligned}
L_{u, r}(v) & \equiv \sigma^{r}\left(\Delta_{u}-1\right)\left(\sigma^{-r} v\right) \\
& =\Delta_{u} v-2 r \sigma^{-1} H_{u}(\sigma, v)+c_{u, r} \cdot v,
\end{aligned}
$$

where

$$
H_{u}(f, g) \equiv \int_{0}^{1}\left(\frac{n \omega_{t}^{n-1} \wedge d f \wedge d^{c} g}{\omega_{t}^{n}}\right) d t
$$

and

$$
\begin{aligned}
c_{u, r} & \equiv \sigma^{r} \Delta_{u}\left(\sigma^{-r}\right)-1, \\
& =r \sigma^{-1} \Delta_{u}(-\sigma)+r(r+1) \sigma^{-2} H_{u}(\sigma, \sigma)-1 .
\end{aligned}
$$

It is easy to show $\sigma^{-1} H_{u}(\sigma, \cdot)$ and $c_{u, r}$ are well defined in each local quasicoordinate chart. Indeed, it suffices to check that $\sigma^{-1} d \sigma$ and $\sigma^{-2} d \sigma \wedge d^{c} \sigma$ are well defined. For a coordinate neighborhood $\left(U,\left\{z^{1}, \ldots, z^{n}\right\}\right)$ of $p \in D$ 
with $U=\cup_{0<\eta<1} \phi_{\eta}\left(V_{\eta}\right)$, assume that $D \cap U=\left\{z^{1}=0\right\}$ and that

$$
|s|^{2}=\left|z^{1}\right|^{2} e^{w}, \quad w \in C^{\infty}(U) .
$$

Then, for each $\left(V_{\eta}, \phi_{\eta}\right)$, one has

$$
\begin{aligned}
& z^{1}=e^{(1+\eta) /(1-\eta) \cdot\left(v^{1}+1\right) /\left(v^{1}-1\right)} \\
& z^{j}=v^{j}, \quad j=2, \ldots, n,
\end{aligned}
$$

on $V_{\eta} \equiv \Delta_{3 / 4} \times \Delta^{n-1}$. These imply that

$$
\frac{d z^{1}}{z^{1} \log \left|z^{1}\right|^{2}}=\frac{\bar{v}^{1}-1}{v^{1}-1} \cdot \frac{d v^{1}}{1-\left|v^{1}\right|^{2}}
$$

which is independent of $\eta$, and so

$$
\frac{d z^{1} \wedge d \bar{z}^{1}}{\left|z^{1}\right|^{2} \log ^{2}\left|z^{1}\right|^{2}}=\frac{d v^{1} \wedge d \bar{v}^{1}}{\left(1-\left|v^{1}\right|^{2}\right)^{2}}
$$

for $0 \leq\left|v^{1}\right| \leq 3 / 4$. Therefore, we have showed that $L_{u, r}$ is uniformly elliptic in each local quasi-coordinate chart and satisfies (3.8).

Next we show that $L_{u, r}$ is an isomorphism. Firstly, we observe that there exists a constant $K_{r}>0$ such that

$$
\sup c_{u, r}<K_{r}
$$

Indeed, one has the more precise estimate as follows: On one hand, there exists a constant $\Lambda>0$ such that

$$
-\Lambda \omega_{K}<\omega_{c}<\Lambda \omega_{K}
$$

We can choose the norm $|\cdot|$ of $s$ sufficiently small such that

$$
\frac{2 \Lambda}{\sigma}<\frac{1}{2}
$$


Then,

$$
\begin{aligned}
\Delta_{u}(-\sigma) & =\int_{0}^{1} \frac{n \omega_{t}^{n-1} \wedge\left(\omega_{c}\right)}{\omega_{t}^{n}} d t \\
& \leq \Lambda \int_{0}^{1} \frac{n \omega_{t}^{n-1} \wedge \omega_{K}}{\omega_{t}^{n}} d t \\
& \leq \frac{n \omega^{n-1} \wedge \omega_{K}}{\omega^{n}} \int_{0}^{1} \frac{d t}{t / C+(1-t)} \\
& \leq 2 \Lambda n C_{1}, \quad C_{1} \equiv \frac{C \ln C}{C-1}>0 .
\end{aligned}
$$

Hence,

$$
r\left(\sigma^{-1}\right) \Delta_{u}(-\sigma) \leq r \frac{2 \Lambda}{\sigma} n C_{1} \leq n r \frac{C_{1}}{2} .
$$

On the other hand, (3.6) implies that

$$
\frac{n \omega_{t}^{n-1} \wedge 2 \sigma^{-2} d \sigma \wedge d^{c} \sigma}{\omega_{t}^{n}} \leq \frac{1}{t / C+(1-t)} \cdot \frac{n \omega^{n-1} \wedge 2 \sigma^{-2} d \sigma \wedge d^{c} \sigma}{\omega^{n}} .
$$

Write

$$
\frac{n \omega^{n-1} \wedge 2 \sigma^{-2} d \sigma \wedge d^{c} \sigma}{\omega^{n}}=\frac{1}{1+f_{b}}
$$

in which

$$
f_{b} \equiv \frac{\left(\omega_{K}+2 \sigma^{-1} \omega_{c}\right)^{n}}{n\left(\omega_{K}+2 \sigma^{-1} \omega_{c}\right)^{n-1} \wedge 2 \sigma^{-2} d \sigma \wedge d^{c} \sigma}>0 .
$$

Hence,

$$
\begin{aligned}
2 \sigma^{-2} H_{u}(\sigma, \sigma) & \leq \frac{1}{1+f_{b}} \int_{0}^{1} \frac{d t}{t / C+(1-t)} \\
& \leq \frac{C \ln C}{C-1} \equiv C_{1}
\end{aligned}
$$

Let

$$
K_{r}=\frac{r(r+1)}{2} C_{1}+\frac{n r}{2} C_{1}>0
$$

therefore, we have

$$
\sup _{M} c_{u, r} \leq K_{r}-1
$$


Secondly, we have the following two lemmas:

Lemma 3.2. $L_{K} \equiv L_{u, r}-K_{r}: C^{k+2, \alpha}(M) \rightarrow C^{k, \alpha}(M)$ is a linear homeomorphism.

Proof of Lemma 3.2. This proof is similar to Cheng-Yau's in [8]. The injectivity of $L_{K}$ follows immediately from Yau's generalized maximum principle $[8$, p. 516]. The surjectivity can be proved as follows. Since $(M, \omega)$ is a complete manifold, we can choose a sequence of relatively compact domains $\left\{B_{j}\right\}_{j=1}^{\infty}$ to exhaust $M$. The standard Schauder theory (see, for example, Gilbarg and Trudinger [17, p. 107].) implies the following Direchlet problem,

$$
\begin{aligned}
L_{K} v=f, & \text { on } B_{j}, \\
v=0, & \text { on } \partial B_{j},
\end{aligned}
$$

has a unique solution $v_{j} \in C^{k+2, \alpha}\left(\overline{B_{j}}\right)$. It follows from (3.13) and the usual maximum principle that

$$
\sup _{\overline{B_{j}}}\left|v_{j}\right| \leq \sup _{M}|f|, \quad \text { for all } j \in \mathbb{N} .
$$

Then the standard interior Schauder estimates (see, for example, [17, p. 93]) applied to the local quasi-coordinates show that a subsequence of $\left\{v_{j}\right\}$ converges to $v \in C^{k+2, \alpha}(M)$, which satisfies

$$
\begin{aligned}
L_{K} v & =f \quad \text { on } M, \\
\|v\|_{k+2, \alpha} & \leq C\|f\|_{k, \alpha},
\end{aligned}
$$

where $C$ is a constant independent of $v$. This proves Lemma 3.2.

Lemma 3.3. Let $L_{u, r}$ be defined as in (3.7). Then either

(1) the homogeneous problem

$$
L_{u, r} v=0
$$

has non-trivial solutions, which form a finite dimensional subspace of $C^{k+2, \alpha}(M)$, or

(2) the inhomogeneous problem

$$
L_{u, r} v=f
$$

has a unique $C^{k+2, \alpha}(M)$ solution for all $f \in C^{k, \alpha}(M)$. 
For a proof of Lemma 3.3, we are going to make use of the standard Fredholm alternative for compact linear mapping.

Theorem 3.4 (Fredholm alternative for compact linear mapping). Let $V$ be a normed linear space and let $T: V \rightarrow V$ be a compact linear mapping. Then either

(1) $\operatorname{ker}(I-T) \neq\{0\}$, and $\operatorname{dim} \operatorname{ker}(I-T)<\infty$; or

(2) $I-T: V \rightarrow V$ is a linear isomorphism.

See, for example, [17, p. 76], for a proof.

Proof of Lemma 3.3. By definition

$$
L_{u, r}=L_{K}+K_{r}
$$

It follows from Lemma 3.2 that $L_{K}$ has a bounded inverse

$$
L_{K}^{-1}: C^{k, \alpha}(M) \longrightarrow C^{k+2, \alpha}(M) .
$$

Now for any $f \in C^{k, \alpha}(M), L_{u, r} v=f$ is equivalent to

$$
v+K_{r} L_{K}^{-1} v=L_{K}^{-1} f .
$$

We claim that $L_{K}^{-1}: C^{k, \alpha}(M) \rightarrow C^{k, \alpha}(M)$ is a bounded compact linear operator. Indeed, this follows from the Ascoli-Arzelà theorem and the following commutative diagram:

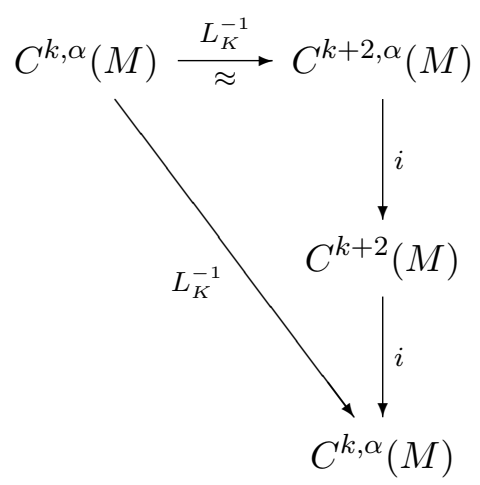

Therefore, by the above theorem of Fredholm alternative, either

$$
v+K_{r} L_{K}^{-1} v=0
$$


has non-trivial solutions which form a finite dimensional subspace of $C^{k, \alpha}(M)$ or, for each $\tilde{f} \in C^{k, \alpha}(M)$, there exists a unique $v \in C^{k, \alpha}(M)$ such that

$$
v+K_{r} L_{K}^{-1} v=\tilde{f}
$$

Note that in the first case, (3.15) implies that $v=-K_{r} L_{K}^{-1} v \in C^{k+2, \alpha}(M)$, and so

$$
\operatorname{ker} L_{u, r}=\operatorname{ker}\left(I+K_{r} L_{K}^{-1}\right)
$$

If ker $L_{u, r}=\{0\}$, then for each $f \in C^{k, \alpha}(M), L_{K}^{-1} f \in C^{k+2, \alpha}(M) \subset C^{k, \alpha}(M)$, there is a unique $v \in C^{k, \alpha}(M)$ that satisfies (3.14), which in turn implies that $v \in C^{k+2, \alpha}(M)$ and that $L_{u, r} v=f$. This finishes the proof of Lemma 3.3.

Finally, note that (3.8) implies $\operatorname{ker} L_{u, r}=\{0\}$. This together with Lemma 3.3 shows that $L_{u, r}$ is a continuous linear isomorphism and hence, a linear homeomorphism. Therefore, the proof of Theorem 3.1 is completed.

Similarly we also have the following linear version of the isomorphism theorem.

Theorem 3.5. Assume that for some $v \in \mathcal{R}(M), \omega_{v} \equiv \omega+d d^{c} v$ satisfies $1 / C \omega \leq \omega_{v} \leq C \omega$ for some $C>0$. Then for each $r \in \mathbb{R}_{+}, k \geq 0$ and $\alpha \in$ $(0,1)$, we have the following commutative diagram:

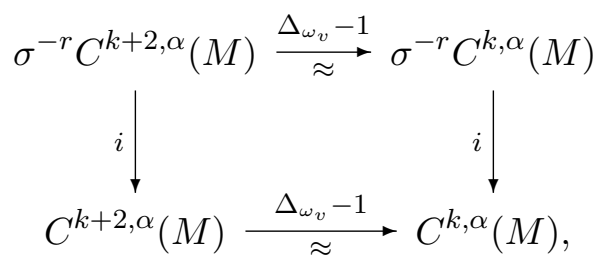

where $\Delta_{\omega_{v}}$ is the negative Laplacian with respect to the metric $\omega_{v}$, and $\approx$ stands for the homeomorphism of the Banach spaces.

The proof is similar but easier. So we omit it here. 


\subsection{Characterization of the solution of Monge-Ampère equations}

Recall the weighted Cheng-Yau Hölder rings $\mathcal{R}_{r}(M), r \geq 0$, introduced in Section 2:

$$
\mathcal{R}(M):=\bigcap_{k \geq 0,0<\alpha<1} C^{k, \alpha}(M)
$$

is the Cheng-Yau Hölder ring. For each $r \in \mathbb{R}_{+}$,

$$
\mathcal{R}_{r}(M):=\sigma^{-r} \mathcal{R}(M)
$$

is the weight $r$ Cheng-Yau Hölder ring, which is an ideal in $\mathcal{R}(M)$. Then any sequence of non-decreasing numbers $\left\{r_{j}\right\}_{j=1}^{\infty} \subset \mathbb{R}_{+}$gives rise to a filtration of $\mathcal{R}(M)$ :

$$
\mathcal{R}_{r_{1}}(M) \supset \mathcal{R}_{r_{2}}(M) \supset \mathcal{R}_{r_{3}}(M) \supset \cdots .
$$

Now before carrying out the asymptotic expansion, we give a precise characterization of the solution by the aid of Theorem 3.1.

Theorem 3.6. If $D \subset \bar{M}$ is a smooth divisor, then one can choose a canonical metric on $D$ such that the solution of

$$
\begin{aligned}
& M_{\omega}(u)=J(\Omega)^{-1}, \\
& \frac{1}{C} \omega \leq \omega_{u} \leq C \omega, \quad C>0 .
\end{aligned}
$$

is in $\mathcal{R}_{1}(M)$.

Proof of Theorem 3.6. It suffices to construct an initial volume form $\Omega$ on $M$ such that

$$
\log J(\Omega) \in \mathcal{R}_{1}(M) .
$$

Given any volume $\widetilde{V}$ on $\bar{M}$ and a metric $h$ on the line bundle $[D], \widetilde{V} / h$ give rise to a metric on $K_{\bar{M}}+[D]$. Without loss of generality, suppose the curvature form

$$
\widetilde{\omega} \equiv \operatorname{Ric}\left(\frac{\widetilde{V}}{h}\right)>0 .
$$

Then, by the adjunction formula, the pull back

$$
c_{1}\left(K_{D}\right) \ni i^{*}(\widetilde{\omega})>0 .
$$


It follows from Yau's solution of Calabi conjecture ([39], see also [1]) that there exists a function $\varphi_{D}$ on $D$ unique up to a constant such that

$$
\omega_{K-E, D} \equiv i^{*}(\widetilde{\omega})+d d^{c} \varphi_{D}
$$

is the Kähler-Einstein metric on $D$ with the Einstein constant -1 .

We claim that $\varphi_{D}$ can be extended to a smooth function $\varphi$ on $\bar{M}$ such that

$$
\left.\varphi\right|_{D}=\varphi_{D}
$$

and

$$
\tilde{\omega}+d d^{c} \varphi>0 \text { on } \bar{M}
$$

We can choose a large constant $C_{D}$ depending only on $D$ such that

$$
\widetilde{\omega}+d d^{c}\left(\varphi_{D}+C_{D}|s|^{2}\right)=\widetilde{\omega}+d d^{c} \varphi_{D}+C_{D} h d z^{1} \wedge d \bar{z}^{1}>0
$$

at every point of $D$. Then there exists a small constant $\delta>0$ such that

$$
\widetilde{\omega}+d d^{c}\left(\varphi_{D}+C_{D}|s|^{2}\right)>0 \text { on }\left\{|s|^{2}<\delta\right\} .
$$

Let $\chi \in C^{\infty}(\mathbb{R})$ be a non-negative cut-off function with $\chi \equiv 1$ on $[-1,1]$ and vanishes outside $[-2,2]$, and let

$$
\varphi_{\chi}=\chi\left(|s|^{2} / \delta\right)\left(\varphi_{D}+C_{D}|s|^{2}\right)
$$

Let

$$
\varphi=\frac{1}{m} \log \left(e^{m \varphi_{x}}+C|s|^{6}\right),
$$

where $m \in \mathbb{N}$ and $C>1$ are constants to be determined. Then we have $\varphi \in C^{\infty}(\bar{M}),\left.\varphi\right|_{D}=\varphi_{D}$, and

$$
\widetilde{\omega}+d d^{c} \varphi=\widetilde{\omega}+d d^{c}\left(\varphi_{D}+C_{D}|s|^{2}\right)>0
$$

at every point of $D$. On $M=\bar{M} \backslash D$, we have

$$
\widetilde{\omega}+d d^{c} \varphi \geq \frac{e^{m \varphi_{\chi}}\left(\widetilde{\omega}+d d^{c} \varphi_{\chi}\right)}{e^{m \varphi_{\chi}}+C|s|^{6}}+\frac{(C / m)|s|^{6}\left(m \widetilde{\omega}+3 d d^{c} \log h\right)}{e^{m \varphi_{\chi}}+C|s|^{6}} .
$$

Since $\widetilde{\omega}>0$, we can choose $m$ sufficiently large such that

$$
m \widetilde{\omega}+3 d d^{c} \log h>0 .
$$

On the region $\left\{|s|^{2} \geq \delta / 2\right\}$, for a fixed $m$, we can choose the constant $C=$ $C(\delta, m)$ sufficiently large such that the second term in (3.20) dominates the 
first term, while on $\left\{0<|s|^{2}<\delta\right\}$, both two terms in (3.20) are positive definite. Therefore, $\widetilde{\omega}+d d^{c} \varphi$ is positive definite on the whole $M$. This together with (3.19) proves the claim.

Now by adding a constant to $\varphi$, we get that

$$
\frac{\left.\left(e^{\varphi} \tilde{\gamma} / h\right)\right|_{\left\{z^{1}=0\right\}}}{\operatorname{det}\left(g_{D, i \bar{j}}\right)}=\frac{1}{2 n !},
$$

where we write

$$
\widetilde{V} \equiv \widetilde{\gamma} \prod_{j=1}^{n}\left(\frac{\sqrt{-1}}{2 \pi} d z^{j} \wedge d \bar{z}^{j}\right)
$$

and

$$
\omega_{K-E, D} \equiv \sum_{2 \leq i, j \leq n} g_{D, i \bar{j}}\left(\frac{\sqrt{-1}}{2 \pi} d z^{i} \wedge d \bar{z}^{j}\right) .
$$

Let $V=e^{\varphi} \widetilde{V}$. Then we define the initial volume form $\Omega$ as in (3.1).

Recall that

$$
\omega=\omega_{K}+2 \sigma^{-1} \omega_{c}+2 \sigma^{-2} d \sigma \wedge d^{c} \sigma .
$$

Then,

$$
\omega^{n}=\left(1+\sum_{k=1}^{n-1} \sigma^{-k} f_{k}\right)\left(1+f_{b}\right) \cdot n \omega_{K}^{n-1} \wedge \sigma^{-2} 2 d \sigma \wedge d^{c} \sigma
$$

where

$$
f_{k}=\frac{2^{k}\left(\begin{array}{c}
n-1 \\
k
\end{array}\right) \omega_{K}^{n-k-1} \wedge \omega_{c}^{k} \wedge 2 \sigma^{-2} d \sigma \wedge d^{c} \sigma}{\omega_{K}^{n-1} \wedge 2 \sigma^{-2} d \sigma \wedge d^{c} \sigma}, \quad k=1, \ldots, n-1
$$

nd $f_{b}$ is defined in (3.12). Then,

$$
\begin{aligned}
J(\Omega) & \equiv \frac{(\operatorname{Ric} \Omega)^{n}}{\Omega} \\
& =\frac{\omega^{n}|s|^{2} \log ^{2}|s|^{2}}{V} \\
& =f_{0}\left(1+\sum_{k=1}^{n-1} \sigma^{-k} f_{k}\right)\left(1+f_{b}\right),
\end{aligned}
$$

where

$$
f_{0}=\frac{2 n \omega_{K}^{n-1} \wedge|s|^{2} d \log |s|^{2} \wedge d^{c} \log |s|^{2}}{V}>0
$$


So, it suffices to show that

$$
f_{0}-1 \in \mathcal{R}_{r}(M), \quad \text { for all } r \geq 1 .
$$

In local coordinate $\left\{U,\left(z^{1}, \ldots, z^{n}\right)\right\}$, set $D \cap U=\left\{z^{1}=0\right\}$. Write

$$
\begin{aligned}
|s|^{2} & =\left|z^{1}\right|^{2} e^{w}, \quad w \in C^{\infty}(U) ; \\
V & =\gamma \prod_{j=1}^{n} \frac{\sqrt{-1}}{2 \pi} d z^{j} \wedge d \bar{z}^{j}, \quad \gamma=\widetilde{\gamma} e^{\varphi}>0 . \\
\omega_{K} & =\sum R_{i \bar{j}} \frac{\sqrt{-1}}{2 \pi} d z^{i} \wedge d \bar{z}^{j} .
\end{aligned}
$$

Then, straightforward computations show that

$$
f_{0}=\frac{2 n !}{\gamma e^{-u}}\left(R^{1 \overline{1}}+z^{1} R^{\overline{1} i} w_{i}+\bar{z}^{1} R^{1 \bar{j}} w_{j}+\left|z^{1}\right|^{2} R^{i \bar{j}} w_{i} w_{\bar{j}}\right),
$$

where $R^{i \bar{j}} R_{k \bar{j}}=\delta_{i j} \operatorname{det}\left(R_{p q}\right)$ and $w_{k} \equiv\left(\partial w / \partial z^{k}\right)$. Denote

$$
H=\gamma e^{-w} .
$$

It follows that

$$
\begin{aligned}
R^{1 \overline{1}} & =\operatorname{det}\left(\left(\frac{\partial^{2}}{\partial z^{i} \partial \bar{z}^{j}} \log H\right)_{i, j \geq 2}\right) \\
& =\operatorname{det}\left(\left(\left.\frac{\partial^{2}}{\partial z^{i} \partial \bar{z}^{j}} \log H\right|_{\left\{z^{1}=0\right\}}\right)_{i, j \geq 2}\right)+z^{1} B+\bar{z}^{1} \bar{B}+\mathcal{O}\left(\left|z^{1}\right|^{2}\right),
\end{aligned}
$$

where $B \in C^{\infty}(U)$. Therefore, to prove (3.22), it suffices to prove that for each $r \geq 1$, the $C^{k, \alpha}(U)$ norm of

$$
\left(\log \left|z^{1}\right|^{2}\right)^{r} \cdot\left(\frac{2 n ! \operatorname{det}\left(\left(\left.\left(\partial^{2} / \partial z^{i} \partial \bar{z}^{j}\right) \log H\right|_{\left\{z^{1}=0\right\}}\right)_{i, j \geq 2}\right)}{\left.H\right|_{\left\{z^{1}=0\right\}}}-1\right)
$$

is uniformly bounded. This, however, follows immediately from the above construction (3.21). We have also shown that the conclusion $u \in \mathcal{R}_{1}(M)$ is independent of the extension of $\omega_{K-E, D}^{n-1}$ to $\bar{M}$.

Remark 3.7. The reader is referred to Theorem 4.8 in Section 4.2 for constructing the initial volume form in a general setting. 


\subsection{Asymptotic expansions}

Recall that the graded Cheng-Yau Hölder ring $\mathcal{R}^{G}(M)$ associated to the $\log$-filtration $\left\{\mathcal{R}_{j}(M)\right\}_{j=0}^{\infty}$ is given by

$$
\begin{aligned}
\mathcal{R}^{G}(M)= & \left\{u \in \mathcal{R}(M) \mid \text { there exist a sequence }\left\{\psi_{j}\right\}_{j=0}^{\infty},\right. \text { where } \\
& \text { not all } \psi_{j} \text { are zero, such that for each } N \in \mathbb{N}, \\
& \left.u-\sum_{j=0}^{N} \psi_{j} \sigma^{-j} \in \mathcal{R}_{N+1}(M) .\right\}
\end{aligned}
$$

Note that $\sigma^{-\alpha} \in \mathcal{R}^{G}(M)$, but $|s|^{\beta},|s|^{\lambda}\left(-\log |s|^{2}\right)^{\mu} \notin \mathcal{R}^{G}(M)$, where $\alpha, \beta$, $\lambda$ and $\mu \in \mathbb{R}_{+}$. Let

$$
\mathcal{R}_{j}^{G}(M)=\mathcal{R}^{G}(M) \cap \mathcal{R}_{j}(M), \quad j \in \mathbb{N} .
$$

Denote $L_{j}=L_{0, j}$ in (3.7), i.e.,

$$
L_{j}(v)=\Delta_{\omega}(v)-2 j \sigma^{-1} H_{\omega}(\sigma, v)+c_{j} v, \quad j \in \mathbb{N},
$$

where $\Delta_{\omega}$ is the negative Laplacian with respect to the metric $\omega$,

$$
\begin{aligned}
& H_{\omega}(f, g)=\frac{n \omega^{n-1} \wedge d f \wedge d^{c} g}{\omega^{n}}, \quad f, g \in C^{1}(M) \\
& c_{j}=j \sigma^{-1} \Delta_{\omega}(-\sigma)+j(j+1) \sigma^{-2} H_{\omega}(\sigma, \sigma)-1 .
\end{aligned}
$$

Now we derive the desired asymptotic expansion in the following theorem.

Theorem 3.8. With the assumptions in Theorem 3.6, the solution $u$ of (3.17) is in $\mathcal{R}_{1}^{G}(M)$. More precisely, there exists a sequence $\left\{\psi_{j}\right\}_{j=1}^{\infty} \subset$ $\mathcal{R}(M)$ such that for any $N \in \mathbb{N}$,

$$
u-\sum_{j=1}^{N} \psi_{j} \sigma^{-j} \in \mathcal{R}_{N+1}(M),
$$

where each $\psi_{j}$ satisfies

$$
L_{j}\left(\psi_{j}\right)=F_{j}, \quad j \in \mathbb{N},
$$


in which

$$
F_{1}=-\frac{2 n(n-1) \omega_{k}^{n-2} \wedge \omega_{c} \wedge\left(2 d \sigma \wedge d^{c} \sigma / \sigma^{2}\right)}{V}
$$

and $F_{j} \in \mathcal{R}(M), j \geq 2$, are given by induction.

Remark 3.9. The coefficients $\psi_{j}$ of the asymptotics are required to satisfy the Elliptic linear second-order PDE (3.25), in contrast to the ODE in the case of pseudoconvex domain [24].

Proof of Theorem 3.8. As in the proof of Theorem 3.6,

$$
\begin{aligned}
J(\Omega) & =f_{0}\left(1+\sum_{k=1}^{n-1} \sigma^{-k} f_{k}\right)\left(1+f_{b}\right) \\
& =1-\sum_{k=1}^{n-1} f_{k} \sigma^{-k}+F_{b},
\end{aligned}
$$

in which

$$
F_{b}=\left[\left(f_{0}-1\right)+f_{b} f_{0}\right]\left(1+\sum_{k=1}^{n-1} \sigma^{-k} f_{k}\right) \in \bigcap_{r \geq 0} \mathcal{R}_{r}(M)
$$

Let $F_{1}=-f_{1}$, and let $\psi_{1} \in \mathcal{R}(M)$ be the unique solution of

$$
L_{1}\left(\psi_{1}\right)=F_{1}
$$

Then $u_{1} \equiv \psi_{1} \sigma^{-1}$ satisfies that

$$
\left(\Delta_{\omega}-1\right)\left(u_{1}\right)=F_{1} \sigma^{-1} .
$$

We want to show

$$
u-u_{1} \in \mathcal{R}_{2}(M)
$$

Let $h_{1}=u_{1}-u$. By Theorem 3.6 we have $u \in \mathcal{R}_{1}(M)$, so $h_{1} \in \mathcal{R}_{1}(M)$. Observe that by construction

$$
\begin{aligned}
\mathcal{R}_{2}(M) & \ni J\left(\mathrm{e}^{u_{1}} \Omega\right)-J\left(\mathrm{e}^{u} \Omega\right) \\
& =M_{\omega_{u}}\left(h_{1}\right)-1 \\
& =\left(1+\Delta_{\omega_{u}} h_{1}+G_{2}^{u}\left(h_{2}\right)+\cdots+G_{n}^{u}\left(h_{1}\right)\right) \mathrm{e}^{-h_{1}}-1,
\end{aligned}
$$


where

$$
G_{i}^{u}\left(h_{1}\right)=\frac{\left(\begin{array}{c}
n \\
i
\end{array}\right) \omega_{u}^{n-i} \wedge\left(d d^{c} h_{1}\right)^{i}}{\omega_{u}^{n}} \in \mathcal{R}_{i}(M), \quad i=2, \ldots, n
$$

Hence,

$$
\left(\Delta_{\omega_{u}}-1\right) h_{1} \in \mathcal{R}_{2}(M) .
$$

Therefore, it follows from Theorem 3.5 that $h_{1} \in \mathcal{R}_{2}(M)$. This proves (3.26).

Now assume that by induction, we have

$$
u_{N-1}=\sum_{i=1}^{N-1} \psi_{i} \sigma^{-i}
$$

such that

$$
\begin{aligned}
u-u_{N-1} & \in \mathcal{R}_{N}(M) . \\
J\left(\mathrm{e}^{u_{N-1}} \Omega\right) & =1-F_{N} \sigma^{-N}, \quad F_{N} \in \mathcal{R}(M) .
\end{aligned}
$$

Then, there exist a $\psi_{N} \in \mathcal{R}(M)$ such that $L_{N} \psi_{N}=F_{N}$. Let

$$
u_{N}=u_{N-1}+\psi_{N} \sigma^{-N} \text {. }
$$

Thus, we have

$$
J\left(\mathrm{e}^{u_{N}} \Omega\right)=1+F_{N+1} \sigma^{-(N+1)}
$$

for some $F_{N+1} \in \mathcal{R}(M)$. Let $h_{N}=u_{N}-u$. Then, $h_{N} \in \mathcal{R}_{N}(M)$. Furthermore,

$$
\begin{aligned}
\mathcal{R}_{N+1}(M) & \ni J\left(\mathrm{e}^{u_{N}} \Omega\right)-J\left(\mathrm{e}^{u} \Omega\right) \\
& =M_{\omega_{u}}\left(h_{N}\right)-1 \\
& =\left(1+\Delta_{\omega_{u}} h_{N}+G_{2}^{u}\left(h_{N}\right)+\cdots+G_{n}^{u}\left(h_{N}\right)\right) e^{-h_{N}}-1,
\end{aligned}
$$

which implies that

$$
\left(\Delta_{\omega_{u}}-1\right) h_{N} \in \mathcal{R}_{N+1}(M),
$$

Hence, $h_{N} \in \mathcal{R}_{N+1}(M)$ by Theorem 3.5. This completes the induction.

\section{Simple normal crossing case}

In this section, the isomorphism theorems are stated and proved in a much general form. We also derive a theorem on the extension of Hermitian metrics with positive curvature. By combining these results with the iteration 
formula, we obtain the asymptotics of Kähler-Einstein volume form near the complete intersections of the irreducible components of the divisor. Furthermore, the obtained asymptotics is canonical in the sense that it is unique up to infinite weight, provided the metrics of the restricted normal bundles are fixed.

\subsection{Isomorphism theorems}

We first generalize Theorems $3.1,3.5$, and 3.8 to the case that $D$ has simple normal crossing. In this case, let $D=\sum_{i=1}^{p} D_{i}$, where the irreducible components $D_{i}$ are smooth and intersect transversely. Let $s_{i} \in H^{0}\left(\bar{M}, \mathcal{O}\left[D_{i}\right]\right)$ define $D_{i}$ and denote by $\sigma_{i}=-\log \left|s_{i}\right|^{2}$. Choose a volume form $V$ on $\bar{M}$ such that

$$
\Omega \equiv \frac{V}{\prod_{i=1}^{p}\left|s_{i}\right|^{2}\left(\log \left|s_{i}\right|^{2}\right)^{2}}
$$

is the initial volume form on $M$ satisfying the properties (2.2) and (2.2) in Section 2. Recall that

$$
\begin{aligned}
\omega & =\operatorname{Ric} \Omega \\
& =\omega_{K}+2 \sum_{i=1}^{p} \sigma_{i}^{-1} \omega_{c_{i}}+2 \sum_{i=1}^{p} \sigma_{i}^{-2} d \sigma_{i} \wedge d^{c} \sigma_{i},
\end{aligned}
$$

where

$$
\begin{aligned}
& \omega_{K}=\operatorname{Ric}\left(\frac{V}{\prod_{i=1}^{p}\left|s_{i}\right|^{2}}\right) \\
& \omega_{c_{i}}=-d d^{c} \sigma_{i} .
\end{aligned}
$$

For any subset $I=\left\{i_{1}, \ldots, i_{k}\right\} \subset\{1, \ldots, p\}$, denote by

$$
\sigma^{-r_{I}}=\sigma_{i_{1}}^{-r_{i_{1}}} \cdots \sigma_{i_{k}}^{-r_{i_{k}}}, \quad \text { for all } r_{I}=\left(r_{i_{1}}, \ldots, r_{i_{k}}\right) \in \mathbb{R}_{+}^{|I|}
$$

Denote $\sigma^{-r}=\sigma^{-r_{I}}$ if $I=\{1, \ldots, p\}$. Note that we implicitly assume $r_{I} \in \mathbb{Z}_{+}^{|I|}$ when we write $\left|r_{I}\right|=l \in \mathbb{Z}_{+}$in the follows.

The weighted Cheng-Yau Hölder spaces $\sigma^{-r} C^{k, \alpha}(M)$ are the Banach spaces defined as usual. For each $l \in \mathbb{N}, \sum_{\left|r_{I}\right|=l} \sigma^{-r_{I}} C^{k, \alpha}(M)$ is defined to be the Cheng-Yau Hölder space of homogeneous weight $l$ associated to the 
index set $I$. Recall that

$$
\begin{aligned}
\mathcal{R}_{I, l}(M) & =\tau_{I}^{l} \mathcal{R}(M) \\
& =\sum_{\left|r_{I}\right|=l} \sigma^{-r_{I}} \mathcal{R}(M), \quad l \in \mathbb{N} .
\end{aligned}
$$

in which

$$
\tau_{I} \equiv\left(\sum_{i \in I} \sigma_{i}^{-2}\right)^{1 / 2}
$$

Now we state and prove the following simple normal crossing version of the isomorphism theorem.

Theorem 4.1. Fix an arbitrary $r \in \mathbb{R}_{+}^{p}, k \geq 0$ and $\alpha \in(0,1)$. Let

$$
\omega_{\varphi} \equiv \omega+d d^{c} \varphi, \quad \varphi \in \mathcal{R}(M)
$$

satisfy $\omega / C_{1}<\omega_{\varphi}<C_{1} \omega, C_{1}>0$. For each $F \in \sigma^{-r} C^{k, \alpha}(M)$, let $u \in$ $C^{k+2, \alpha}(M)$ be the unique solution of

$$
\begin{aligned}
M_{\omega_{\varphi}}(u) & \equiv \frac{\left(\omega_{\varphi}+d d^{c} u\right)^{n}}{\omega_{\varphi}} e^{-u}=e^{F}, \\
\frac{1}{C} \omega_{\varphi} & \leq \omega_{\varphi}+d d^{c} u \leq C \omega_{\varphi}, \quad C>0 .
\end{aligned}
$$

Then, $u \in \sigma^{-r} C^{k+2, \alpha}(M)$. Furthermore, for any subset $I \subset\{1, \ldots, p\}$, if

$$
F \in \sum_{\left|r_{I}\right|=l, r_{I} \in \mathbb{Z}_{+}^{|I|}} \sigma^{-r_{I}} C^{k, \alpha}(M), \quad l \in \mathbb{N},
$$

then

$$
u \in \sum_{\left|r_{I}\right|=l, r_{I} \in \mathbb{Z}_{+}^{|I|}} \sigma^{-r_{I}} C^{k+2, \alpha}(M) .
$$

Proof of Theorem 4.1. Firstly, observe that

$$
\sigma^{r} d \sigma^{-r}=-\sum_{i=1}^{p} \frac{r_{i}}{\sigma_{i}} d \sigma_{i}
$$


and

$$
\begin{aligned}
\sigma^{r} d d^{c}\left(\sigma^{-r}\right)= & \sum_{i=1}^{p}\left[-\frac{r_{i}}{\sigma_{i}} d d^{c} \sigma_{i}+\frac{r_{i}\left(r_{i}+1\right)}{\sigma_{i}^{2}} d \sigma_{i} \wedge d^{c} \sigma_{i}\right. \\
& \left.+\sum_{j \neq i} \frac{r_{i} r_{j}}{\sigma_{i} \sigma_{j}} d \sigma_{j} \wedge d^{c} \sigma_{i}\right]
\end{aligned}
$$

Similar to the proof of Theorem 3.1, we define

$$
\begin{aligned}
L_{u, r}(v) & \equiv \sigma^{r}\left(\Delta_{u}-1\right)\left(\sigma^{-r} v\right) \\
& =\Delta_{u} v-2 \sum_{i=1}^{p} r_{i} \sigma_{i}^{-1} H_{u}\left(\sigma_{i}, v\right)+c_{u, r} v
\end{aligned}
$$

where $\Delta_{u}$ and $H_{u}$ are defined by the same form of (3.4) and (3.10) with $\omega_{t}$ replaced by $\omega_{\varphi, t} \equiv \omega_{\varphi}+t d d^{c} u$, respectively; and

$$
\begin{aligned}
c_{u, r} & \equiv \sigma^{r} \Delta_{u}\left(\sigma^{-r}\right)-1, \\
& =\sum_{i=1}^{p}\left[\frac{r_{i}}{\sigma_{i}} \Delta_{u}\left(-\sigma_{i}\right)+\frac{r_{i}\left(r_{i}+1\right)}{\sigma_{i}^{2}} H_{u}\left(\sigma_{i}, \sigma_{i}\right)+\sum_{j \neq i} \frac{r_{i} r_{j}}{\sigma_{i} \sigma_{j}} H_{u}\left(\sigma_{j}, \sigma_{i}\right)\right]-1 .
\end{aligned}
$$

Similarly, $\Delta_{u}, \sigma_{i}^{-1} H_{u}\left(\sigma_{i}, \cdot\right)$ and $c_{u, r}$ are well defined in each local quasicoordinate chart. These follows from the well-definedness of $\sigma_{i}^{-1} d \sigma_{i}$ for $i=1, \ldots, p$ : Without loss of generality, we assume that locally $D \cap U=$ $\left\{z^{1} \cdots z^{k}=0\right\}$ and that

$$
\left|s_{j}\right|^{2}=\left|z^{j}\right|^{2} e^{w_{j}}, \quad w_{j} \in C^{\infty}(U), \quad j=1, \ldots, k .
$$

Let $\left\{\left(V_{\eta}, \phi_{\eta}\right) \mid \eta \in(0,1)^{k}\right\}$ be a family of local quasi-coordinates which covers $U$. Then for each $\left(V_{\eta}, \phi_{\eta}\right)$ in the family, we have

$$
\begin{aligned}
z^{i} & =e^{\left(1+\eta^{i}\right) /\left(1-\eta^{i}\right) \cdot\left(v^{i}+1\right) /\left(v^{i}-1\right)}, \quad i=1, \ldots, k, \\
z^{j} & =v^{j}, \quad j=k+1, \ldots, n,
\end{aligned}
$$

on $V_{\eta}=\Delta_{3 / 4}^{k} \times \Delta^{n-k}$. These imply that

$$
\frac{d z^{i}}{z^{i} \log \left|z^{i}\right|^{2}}=\frac{\bar{v}^{i}-1}{v^{i}-1} \cdot \frac{d v^{i}}{1-\left|v^{i}\right|^{2}},
$$


which is independent of $\eta$, for $0 \leq\left|v^{i}\right| \leq 3 / 4, i=1, \ldots, k$. Therefore, we have shown that $L_{u, r}$ is uniformly elliptic in each local quasi-coordinate chart and satisfies (3.8).

Next, we will derive the following estimate

$$
\sup _{M} c_{u, r} \leq K_{r}-1
$$

where

$$
K_{r}=\frac{1}{2} n C_{2} \sum_{i=1}^{p} r_{i}+2 n C_{2} \sum_{i=1}^{p} r_{i}\left(2 r_{i}+1\right)>0
$$

in which

$$
C_{2}=\frac{C_{1} C \ln C}{(C-1)}
$$

There exists a constant $\Lambda>0$ such that

$$
-\Lambda \omega_{K}<\omega_{c_{i}}<\Lambda \omega_{K}
$$

Also, we can choose the norm $|\cdot|$ of $s$ sufficiently small such that

$$
\Lambda \sum_{i=1}^{p} \frac{2}{\sigma_{i}}<\frac{1}{2}
$$

Note that for all $t \in[0,1]$,

$$
\left[\frac{t}{C}+(1-t)\right] \frac{\omega}{C_{1}} \leq \omega_{t}=\omega_{\varphi}+t d d^{c} u \leq C_{1}[C t+(1-t)] \omega
$$

Then it follows from the same estimate in (3.11) that

$$
\sum_{i=1}^{p} r_{i} \frac{\Delta_{u}\left(-\sigma_{i}\right)}{\sigma_{i}} \leq \frac{n}{2}|r| C_{2}
$$

where $C_{2}>0$ is given by (4.3). Moreover, we have

$$
\sum_{i \neq j}\left(\frac{r_{i} d \sigma_{i}}{\sigma_{i}} \wedge \frac{r_{j} d^{c} \sigma_{j}}{\sigma_{j}}\right) \leq \sum_{k=1}^{p} \frac{r_{k}^{2} d \sigma_{k} \wedge d^{c} \sigma_{k}}{\sigma_{k}^{2}}
$$


and hence,

$$
\sum_{j \neq i} \frac{r_{i} r_{j}}{\sigma_{i} \sigma_{j}} H_{u}\left(\sigma_{j}, \sigma_{i}\right) \leq \frac{r_{i}^{2}}{\sigma_{i}^{2}} H_{u}\left(\sigma_{i}, \sigma_{i}\right)
$$

It remains to control $\sum r_{i}\left(r_{i}+1\right) \sigma_{i}^{-2} H_{u}\left(\sigma_{i}, \sigma_{i}\right)$ : fix an arbitrary $i=1, \ldots, p$,

$$
\frac{n \omega_{t}^{n-1} \wedge 2 \sigma_{i}^{-2} d \sigma_{i} \wedge d^{c} \sigma_{i}}{\omega_{t}^{n}} \leq \frac{C_{1}}{t / C+(1-t)} \cdot \frac{n \omega^{n-1} \wedge 2 \sigma_{i}^{-2} d \sigma_{i} \wedge d^{c} \sigma_{i}}{\omega^{n}} .
$$

Write

$$
\begin{aligned}
\omega^{n}=\left(2 \sigma_{i}^{-2} d \sigma_{i} \wedge d^{c} \sigma_{i}\right) \wedge \omega^{n-1} \\
\quad+\left(\omega_{K}+2 \sum_{i=1}^{p} \sigma_{i}^{-1} \omega_{c_{i}}+2 \sum_{j \neq i} \sigma_{i}^{-2} d \sigma_{i} \wedge d^{c} \sigma_{i}\right) \wedge \omega^{n-1}
\end{aligned}
$$

Then,

$$
\frac{\omega^{n-1} \wedge 2 \sigma_{i}^{-2} d \sigma_{i} \wedge d^{c} \sigma_{i}}{\omega^{n}}=\frac{1}{1+f_{b i}}
$$

in which

$$
f_{b i} \equiv \frac{\left(\omega_{K}+2 \sum_{i=1}^{p} \sigma_{i}^{-1} \omega_{c_{i}}+2 \sum_{j \neq i} \sigma_{i}^{-2} d \sigma_{i} \wedge d^{c} \sigma_{i}\right) \wedge \omega^{n-1}}{\left(2 \sigma_{i}^{-2} d \sigma_{i} \wedge d^{c} \sigma_{i}\right) \wedge \omega^{n-1}}>0 .
$$

Hence,

$$
\sum_{i=1}^{p} r_{i}\left(r_{i}+1\right) \sigma_{i}^{-2} H_{u}\left(\sigma_{i}, \sigma_{i}\right) \leq n C_{2} \sum_{i=1}^{p} r_{i}\left(r_{i}+1\right) .
$$

Therefore, (4.2) follows from (4.4), (4.5) and (4.6).

Then, by going through the same process in the proof of Theorem 3.1, we show that $L_{u, r}: C^{k+2, \alpha}(M) \rightarrow C^{k, \alpha}(M)$ is a linear homeomorphism. This proves the first part of the theorem.

For the second part, we write

$$
F=\sum_{\left|r_{I}\right|=l} F_{r_{I}}, \quad F_{r_{I}} \in \sigma^{-r_{I}} C^{k, \alpha}(M) .
$$

Denote by $u \in C^{k+2, \alpha}(M)$ the corresponding solution for $F$. Each $r_{I} \in \mathbb{Z}_{+}^{|I|}$ can be viewed as an element in $\mathbb{R}_{+}^{p}$ via the natural embedding 
$I \hookrightarrow\{1, \ldots, p\}$; hence, by the argument above, we know that

$$
\Delta_{u}-1: \sigma^{-r_{I}} C^{k+2, \alpha}(M) \rightarrow \sigma^{-r_{I}} C^{k, \alpha}(M)
$$

is a linear homeomorphism for each $r_{I} \in \mathbb{Z}_{+}^{|I|}$. Then, there exists a $u_{r_{I}} \in$ $\sigma^{-r_{I}} C^{k+2, \alpha}(M)$ such that

$$
\left(\Delta_{u}-1\right)\left(u_{r_{I}}\right)=F_{r_{I}}, \quad \text { for all }\left|r_{I}\right|=l
$$

Hence, by linearity,

$$
\left(\Delta_{u}-1\right)\left(\sum_{\left|r_{I}\right|=l} u_{r_{I}}\right)=F .
$$

Therefore, it follows from the injectivity of $\left(\Delta_{u}-1\right): C^{k+2, \alpha}(M) \rightarrow C^{k, \alpha}(M)$ that

$$
u=\sum_{\left|r_{I}\right|=l} u_{r_{I}}
$$

This proves the second part.

Theorem 4.1 also has a similar linear version, which can be stated in the following:

Theorem 4.2. Let $\omega_{v} \equiv \omega+d d^{c} v$ satisfy $(1 / C) \omega \leq \omega_{v} \leq C \omega$ for some $C>$ 0 , where $v \in \mathcal{R}(M)$. Then, for each $r \in \mathbb{R}_{+}^{p}, k \geq 0$ and $\alpha \in(0,1)$, we have the following commutative diagram:

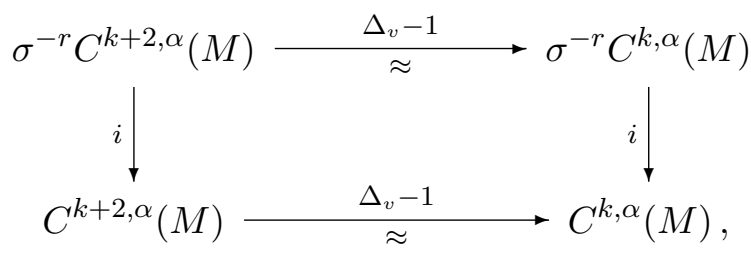

where $\Delta_{\omega_{v}}$ is the negative Laplacian with respect to the metric $\omega_{v}$, and $\approx$ stands for the linear isomorphism of the Banach spaces. Consequently, for 
any $I \subset\{1, \ldots, p\}$, let $r_{I} \in \mathbb{Z}_{+}^{|I|}, l \in \mathbb{N} ;$ we have

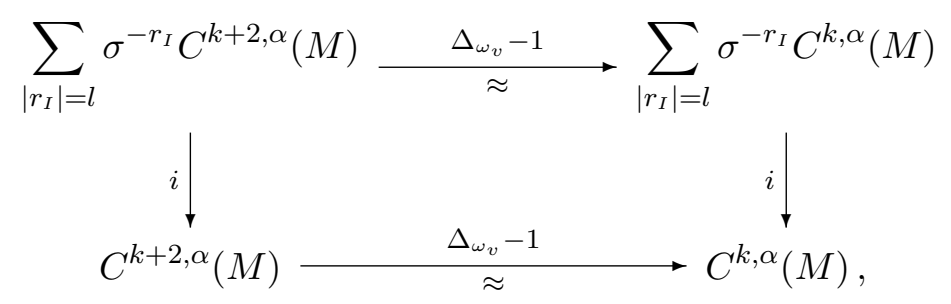

\subsection{Canonical initial volume forms and the metric extension theorem}

Based on these two isomorphism theorems, we can derive the asymptotics of the Kähler-Einstein metric near each connected component of the nonempty complete intersection $D_{I}$, where $I \in \mathcal{I}$. ( $\mathcal{I}$ is defined in Section 2.2.) Without loss of generality, assume that the index set $I=\{1, \ldots, q\}$. Let

$$
D_{I}=D_{\{1, \ldots, q\}}=\sum_{\nu} D_{I}^{\nu}
$$

where each $D_{I}^{\nu}$ is a connected component.

Observe that by repeatedly using the adjunction formula, we have

$$
\left.\left(K_{\bar{M}}+[D]\right)\right|_{D_{I}^{\nu}}=K_{D_{I}^{\nu}}
$$

for each component $D_{I}^{\nu}$. Then, it follows from the positivity condition (1.2) and Yau's solution of Calabi conjecture that there exists a unique KählerEinstein metric $\omega_{K-E, \nu}$ of Ricci curvature -1 on $D_{I}^{\nu}$, when $|I|=q<n$. In the case $q=n$, each $D_{I}^{\nu}$ is just a point in $\bar{M}$; we set $\omega_{K-E, \nu}=1$.

Note that the holomorphic defining section of a divisor on $\bar{M}$ is unique only up to a constant function on $\bar{M}$. Let us first fix one of them. Let

$$
S_{I}=\left\{s_{i} \in H^{0}\left(\bar{M}, \mathcal{O}\left(\left[D_{i}\right]\right) \mid\left(s_{i}\right)=D_{i} \quad \text { for } i \in I=\{1, \ldots, q\}\right\}\right.
$$

be a set of given defining sections, and let

$$
H_{I}=\left\{h_{I, i} \mid i \in I=\{1, \ldots, q\}\right\}
$$


where each $h_{I, i}$ is an arbitrary given metric on the restriction of the normal bundle $\left.N_{D_{i}}\right|_{D_{I}}=\left.\left[D_{i}\right]\right|_{D_{I}}$. Since $D_{j} \cap D_{I}=\emptyset$ for $j \notin I$, we have

$$
\left(\prod_{j=q+1}^{p} \sigma_{i}^{2}\right)^{-1} \in C^{\infty}\left(D_{I}\right)
$$

In order to derive an asymptotic expansion near $D_{I}$ with certain canonicity, we introduce the family, $\mathcal{M}_{I ; S_{I}, H_{I}}$, of initial volume forms

$$
\Omega=\frac{V}{\prod_{i=1}^{p}\left|s_{i}\right|^{2}\left(\log \left|s_{i}\right|^{2}\right)^{2}},
$$

satisfy the following constraints:

(1) For each connected component $D_{I}^{\nu}$,

$$
\left.\frac{\gamma}{\prod_{i \in I} h_{i} \prod_{j \notin I}\left|s_{j}\right|^{2} \sigma_{j}^{2}}\right|_{D_{I}^{\nu}}= \begin{cases}2^{q} n ! \operatorname{det}\left(g_{i \bar{j}, \nu}\right), & \text { if } 1 \leq|I|<n ; \\ 2^{n} n !, & \text { if }|I|=n .\end{cases}
$$

(2) $s_{i} \in S_{I}$ for $i \in I$. (For $j \notin I, s_{j}$ is unique only up to a constant.)

(3) Each $\left.h_{i}\right|_{D_{I}}=h_{I, i}$, for any $i \in I=\{1, \ldots, q\}$, where locally $D_{I}^{\nu}$ is given by $\left\{z^{1} \cdots z^{q}=0\right\}$ and denote

$$
\begin{aligned}
V & =\gamma \prod_{j=1}^{n}\left(\frac{\sqrt{-1}}{2 \pi} d z^{j} \wedge d \bar{z}^{j}\right), \\
\omega_{K-E, \nu} & =\sum_{q+1 \leq i, j \leq n} g_{i \bar{j}, \nu}\left(\frac{\sqrt{-1}}{2 \pi} d z^{i} \wedge d \bar{z}^{j}\right),
\end{aligned}
$$

and $h_{i}$ is the metric on $\left[D_{i}\right]$ such that $\left|s_{i}\right|^{2}=h_{i}\left|z^{i}\right|^{2}, i=1, \ldots, q$. Given an index set $I$, we say that two families $\mathcal{M}_{I ; S_{I}, H_{I}}$ and $\mathcal{M}_{I ; S_{I}^{\prime}, H_{I}^{\prime}}$ are canonically compatible if

$$
\frac{h_{I, i}}{h_{I, i}^{\prime}}=\left|\frac{s_{i}^{\prime}}{s_{i}}\right|^{2} \equiv \text { constant }
$$

where $h_{I, i} \in H_{I}, h_{I, i}^{\prime} \in H_{I}^{\prime}, s_{i} \in S_{I}$ and $s_{i}^{\prime} \in S_{I}^{\prime}$, for each $i \in I$. Note that (4.9) is nothing but

$$
\left.\frac{\left|s_{i}\right|^{2}}{\left|s_{i}^{\prime}\right|^{\prime 2}}\right|_{D_{I}} \equiv 1 \quad \text { for each } i \in I,
$$

where $|\cdot|^{\prime}$ is the metric on $\left[D_{i}\right]$ locally given by $h_{i}^{\prime}$. 
It is easy to see that the canonical compatibility is an equivalence relation. In particular, $\mathcal{M}_{I ; S_{I}, H_{I}}$ is canonically compatible with itself. Let $\mathcal{M}_{I, \mathcal{H}_{I}}$ be the union of all the families $\mathcal{M}_{I ; S_{I}^{\prime}, H_{I}^{\prime}}$ which are canonically compatible with a given $\mathcal{M}_{I ; S_{I}, H_{I}}$. Finally, for convenience, we denote by $\mathcal{M}_{I}$ the family of initial volume forms which satisfies condition (4.2) only. Before actually constructing such a family, we make the following remark.

Remark 4.3. The initial volume form together with property (4.2) only enable us to obtain an asymptotics whose coefficients are all given by the solutions of certain second-order linear elliptic PDE (see the proof of Theorem 4.12), similar to Theorem 3.8. The additional compatibility condition, however, will assure that the obtained asymptotics, with respect to $\mathcal{R}^{G}(M)$, is canonical in the sense of the following two propositions:

Proposition 4.4. Let $\Omega, \Omega^{\prime} \in \mathcal{M}_{I, \mathcal{H}_{I}}$, and $\omega=\operatorname{Ric}(\Omega), \omega^{\prime}=\operatorname{Ric}\left(\Omega^{\prime}\right)$. Let $u$ and $u^{\prime}$ be the solution of

$$
\begin{aligned}
& M_{\omega}(u)=J(\Omega)^{-1}, \quad \frac{1}{C} \omega<\omega+d d^{c} u<C \omega, \quad C>0 ; \\
& M_{\omega^{\prime}}\left(u^{\prime}\right)=J\left(\Omega^{\prime}\right)^{-1}, \quad \frac{1}{C^{\prime}} \omega^{\prime}<\omega^{\prime}+d d^{c} u<C^{\prime} \omega^{\prime}, \quad C^{\prime}>0,
\end{aligned}
$$

respectively. Then, $u-u^{\prime} \in \mathcal{R}_{I, \infty}$.

Proof of Proposition 4.4. By definition, we have

$$
J\left(e^{u} \Omega\right)=1=J\left(e^{u^{\prime}} \Omega^{\prime}\right) .
$$

By the uniqueness of Kähler-Einstein volume form on $M$,

$$
e^{u} \Omega=e^{u^{\prime}} \Omega^{\prime}=\Omega_{K-E, M}
$$

Observe that

$$
\frac{\Omega^{\prime}}{\Omega}=G \prod_{i=1}^{q}\left(\frac{\log \left|s_{i}\right|^{2}}{\log \left|s_{i}^{\prime}\right|^{\prime 2}}\right)^{2}
$$

where

$$
G=\left(\frac{V^{\prime}}{V} \prod_{i=1}^{p} \frac{\left|s_{i}\right|^{2}}{\left|s_{i}^{\prime}\right|^{\prime 2}} \prod_{j=q+1}^{p} \frac{\log \left|s_{i}\right|^{2}}{\log \left|s_{i}^{\prime}\right|^{\prime 2}}\right) \in \mathcal{R}(M) .
$$

Also, $G$ is smooth near each $D_{I}^{\nu}$; furthermore, by condition (1), we have

$$
\left.G\right|_{D_{I}^{\nu}}=1
$$


for each $\nu$. Hence, $G \in 1+\mathcal{R}_{I, \infty}(M)$. On the other hand,

$$
-\log \left|s_{i}\right|^{2}=\sigma_{i}=\sigma_{i}^{\prime}+\log \beta_{i}=\sigma_{i}^{\prime}\left(1+\sigma_{i}^{\prime-1} \log \beta_{i}\right),
$$

where $\beta_{i}=\left|s_{i}\right|^{2} /\left|s_{i}^{\prime}\right|^{\prime 2} \in C^{\infty}(\bar{M})$. Since $\left.\beta_{i}\right|_{D_{I}}=1$, we have $\beta_{i} \in 1+\mathcal{R}_{I, \infty}(M)$ and $\sigma_{i}^{\prime-1} \log \beta_{i} \in \mathcal{R}_{I, \infty}(M)$. Therefore,

$$
u-u^{\prime}=\log \frac{\Omega^{\prime}}{\Omega} \in \mathcal{R}_{I, \infty}(M) .
$$

Our asymptotics will be derived by the following linear operator $L_{r}$ associated with $\omega$ :

$$
L_{r}(v) \equiv \Delta_{\omega} v-2 \sum_{i=1}^{q} r_{i} \sigma_{i}^{-1} H\left(\sigma_{i}, v\right)+c_{r} v=-f_{r},
$$

where $\Delta_{\omega}$ is the negative Laplacian with respect to $\omega$, and

$$
\begin{aligned}
H(v, w) & =\frac{n \omega^{n-1} \wedge d w \wedge d^{c} v}{\omega^{n}}, \\
c_{r} & =\sum_{i=1}^{q}\left[\frac{r_{i}}{\sigma_{i}} \Delta_{\omega}\left(-\sigma_{i}\right)+\frac{r_{i}\left(r_{i}+1\right)}{\sigma_{i}^{2}} H\left(\sigma_{i}, \sigma_{i}\right)+\sum_{j \neq i} \frac{r_{i} r_{j}}{\sigma_{i} \sigma_{j}} H\left(\sigma_{j}, \sigma_{i}\right)\right]-1 .
\end{aligned}
$$

Proposition 4.5. Let $\Omega, \Omega^{\prime} \in \mathcal{M}_{I, \mathcal{H}_{I}}$, and $\omega=\operatorname{Ric}(\Omega), \omega^{\prime}=\operatorname{Ric}\left(\Omega^{\prime}\right)$. Consider the the following two equations:

$$
\begin{aligned}
L_{r} v & =f, \\
L_{r}^{\prime} v^{\prime} & =f^{\prime},
\end{aligned}
$$

where $v, v^{\prime} \in \mathcal{R}(M)$. If $f-f^{\prime} \in \mathcal{R}_{I, \infty}(M)$, then $v-v^{\prime} \in \mathcal{R}_{I, \infty}(M)$.

To prove this proposition, we first observe the following isomorphic property of the operator $L_{r}$ :

Lemma 4.6. Let $v \in C^{k+2, \alpha}(M)$ satisfy $L_{r}(v)=f$. If

$$
f \in \tau_{I}^{m} C^{k, \alpha}(M), \quad m \in \mathbb{N},
$$

then

$$
v \in \tau_{I}^{m} C^{k+2, \alpha}(M) .
$$

Consequently, if $f \in \mathcal{R}_{I, \infty}(M)$, then $v \in \mathcal{R}_{I, \infty}(M)$. 
Proof of Lemma 4.6. Recall that

$$
\begin{aligned}
\tau_{I} & =\left(\sum_{i \in I} \sigma_{i}^{-2}\right)^{1 / 2} \\
& =\sum_{i \in I}\left(\frac{\sigma_{i}}{\tau_{I}}\right) \sigma_{i} \in \sum_{i \in I} \sigma_{i} \mathcal{R}(M)
\end{aligned}
$$

Then,

$$
\tau_{I}^{m} C^{l, \beta}(M)=\sum_{\left|r_{I}\right|=m} \sigma^{-r_{I}} C^{l, \beta}(M), \quad \text { for all } l \in \mathbb{Z}_{+}, \quad \beta \in(0,1) .
$$

Let $f=\sum_{\left|r_{I}\right|=m} \sigma^{-r_{I}} f_{r_{I}}, f_{r_{I}} \in C^{k, \alpha}(M)$. Then,

$$
\left(\Delta_{\omega}-1\right)\left(v \sigma^{-r}\right)=\sum_{\left|r_{I}\right|=m} \sigma^{-r} \sigma^{-r_{I}} f_{I}
$$

On the other hand, it follows from Theorem 4.2 that for each $r_{I}$, there exists a $v_{r_{I}} \in C^{k+2, \alpha}(M)$ such that

$$
\left(\Delta_{\omega}-1\right)\left(v_{r_{I}} \sigma^{r_{I}} \sigma^{-r}\right)=f_{r_{I}} \sigma^{-r_{I}} \sigma^{-r} .
$$

Thus, by the uniqueness of $\Delta_{\omega}-1$, we have

$$
v=\sum_{\left|r_{I}\right|=m} \sigma^{-r_{I}} v_{r_{I}} \in \tau_{I}^{m} C^{k+2, \alpha}(M)
$$

Next, we derive the following lemma:

Lemma 4.7. Let $\Omega, \Omega^{\prime} \in \mathcal{M}_{I, \mathcal{H}_{I}}$, and $L_{r}$ and $L_{r}^{\prime}$ be the corresponding linear operators with respect to $\Omega$ and $\Omega^{\prime}$, respectively. Then

$$
\left(L_{r}-L_{r}^{\prime}\right)(v) \in \mathcal{R}_{I, \infty}(M), \quad \text { for all } v \in \mathcal{R}(M) .
$$

Proof of Lemma 4.7. We want to show that

$$
\begin{array}{rlrl}
\left(\Delta_{\omega}-\Delta_{\omega^{\prime}}\right) v & \in \mathcal{R}_{I, \infty}(M), & & \text { for all } v \in \mathcal{R}(M), \\
\sigma_{i}^{\prime-1} H\left(\sigma_{i}^{\prime}, v\right)-\sigma_{i}^{-1} H\left(\sigma_{i}, v\right) & \in \mathcal{R}_{I, \infty}(M), & \text { for all } v \in \mathcal{R}(M), \\
c_{r}-c_{r}^{\prime} & \in \mathcal{R}_{I, \infty}(M) . & &
\end{array}
$$


It suffices to check the following:

$$
\begin{aligned}
d \mathcal{R}_{I, l}^{m}(M) & \subset \mathcal{R}_{I, l}^{m+1}(M), \quad \text { for all } l, m \geq 0, \\
\omega^{k}-\omega^{\prime k} & \in \mathcal{R}_{I, \infty}^{k, k}(M), \quad \text { for all } k \geq 1, \\
\sigma_{i}^{-1} d \sigma_{i}-\sigma_{i}^{\prime-1} d \sigma_{i}^{\prime} & \in \mathcal{R}_{I, \infty}^{1}(M) .
\end{aligned}
$$

Recall that

$$
\mathcal{R}_{I, l}^{m}(M)=\tau_{I}^{l} \mathcal{R}^{m}(M)=\sum_{\left|r_{I}\right|=l} \sigma^{-r_{I}} \mathcal{R}^{m}(M) .
$$

For $\varphi \in \mathcal{R}^{m}(M)$, we have

$$
\sigma^{r_{I}} d\left(\sigma^{-r_{I}} \varphi\right)=d \varphi+\sum_{i \in I} r_{i} \frac{d \sigma_{i}}{-\sigma_{i}} \wedge \varphi \in \mathcal{R}^{m+1}(M)
$$

This proves (4.11). Similarly, we have

$$
d^{c} \mathcal{R}_{I, l}^{m}(M) \subset \mathcal{R}_{I, l}^{m+1}(M) .
$$

Next, it follows from (4.11), (4.14) and the proof of Proposition 4.4 that

$$
\omega-\omega^{\prime}=d d^{c} \log \frac{\Omega}{\Omega^{\prime}} \in \mathcal{R}_{I, \infty}^{1,1}(M) .
$$

Since $\omega \in \mathcal{R}^{1,1}(M)$, we get

$$
\omega^{k}-\omega^{\prime k} \in \sum_{i=1}^{k} \omega^{i} \wedge\left(\mathcal{R}_{I, \infty}^{1,1}(M)\right)^{k-i} \subset \mathcal{R}_{I, \infty}^{k, k}(M) .
$$

Finally, as in the proof of Proposition 4.4,

$$
\frac{\sigma_{i}}{\sigma_{i}^{\prime}} \in 1+\mathcal{R}_{I, \infty}(M)
$$

therefore, by (4.11),

$$
\sigma_{i}^{-1} d \sigma_{i}-\sigma_{i}^{\prime-1} d \sigma_{i}^{\prime}=d \log \frac{\sigma_{i}}{\sigma_{i}^{\prime}} \in \mathcal{R}_{I, \infty}^{1}(M)
$$

This completes the proof.

Proof of Proposition 4.5. By the assumption and Lemma 4.7, we have

$$
L_{r}\left(v-v^{\prime}\right)=\left(f-f^{\prime}\right)-\left(L_{r}-L_{r}^{\prime}\right) v^{\prime} \in \mathcal{R}_{I, \infty}(M) .
$$

Hence, it follows from Lemma 4.6 that $v-v^{\prime} \in \mathcal{R}_{I, \infty}(M)$. 
Now given $I=\{1, \ldots, q\}$, we will construct a family $\mathcal{M}_{I ; S_{I}, H_{I}}$ with certain canonicity on the $H_{I}$ : Fix a Kähler metric $\omega_{\bar{M}}$ on $\bar{M}$. Write

$$
\begin{array}{r}
\omega_{M}^{n}=\widetilde{\gamma} \prod_{j=1}^{n}\left(\frac{\sqrt{-1}}{2 \pi} d z^{j} \wedge d \bar{z}^{j}\right) \\
\left(\iota_{i}^{*} \omega_{\bar{M}}\right)^{n-1}=\widetilde{\gamma}_{i} \prod_{j \neq i}\left(\frac{\sqrt{-1}}{2 \pi} d z^{j} \wedge d \bar{z}^{j}\right),
\end{array}
$$

where $\iota_{i}: D_{i} \hookrightarrow \bar{M}$ is the inclusion. Then it follows from the usual adjunction formula that $\bar{M}$ induces a metric $h_{D_{i}}$ on the normal bundle $N_{D_{i}}=$ $\left.\left[D_{i}\right]\right|_{D_{i}}$ :

$$
h_{D_{i}}=\frac{\left.\widetilde{\gamma}\right|_{D_{i}}}{\widetilde{\gamma}_{i}}, \quad i=1, \ldots, q
$$

Now set

$$
h_{I, i}=\left.\epsilon_{i} h_{D_{i}}\right|_{D_{I}}, \quad i=1, \ldots, q .
$$

where each $\epsilon_{i}$ is a small constant. Let

$$
H_{I}=\left\{h_{I, i} \mid i \in I\right\}
$$

This implies that $H_{I}$ is canonical in terms of $\omega_{\bar{M}}$ up to a constant. Extend each $h_{I, i}$ to a smooth metric on $\left[D_{i}\right] \rightarrow \bar{M}$, which is denoted by $h_{i}$.

For each $j \notin I,\left.\left[D_{j}\right]\right|_{D_{I}}$ is a trivial line bundle on $D_{I}$ since $D_{j} \cap D_{I}=\emptyset$. Hence, we can choose a trivial metric on $\left.\left[D_{j}\right]\right|_{D_{I}}$ and extend it to a smooth metric $h_{j}$ on $\left[D_{j}\right] \rightarrow \bar{M}$. By this construction, $\left|s_{j}\right|^{2}$ is a constant on $D_{I}$ for each $j \notin I$.

Note that

$$
\left[d d^{c} \log \frac{\widetilde{\gamma}}{h_{1} \cdots h_{p}}\right]=c_{1}\left(K_{\bar{M}}+[D]\right)
$$

We claim that there exists a function $\rho \in C^{\infty}(\bar{M})$ such that

$$
\begin{aligned}
&\left.\frac{e^{\rho} \widetilde{\gamma}}{\prod_{i=1}^{q} h_{i} \prod_{j=q+1}^{p}\left|s_{j}\right|^{2} \sigma_{j}^{2}}\right|_{D_{I}^{\nu}}= \begin{cases}2^{q} n ! \operatorname{det}\left(g_{i \bar{j}, \nu}\right), & \text { if } 1 \leq q<n \\
2^{n} n !, & \text { if } q=n .\end{cases} \\
& d d^{c} \log \frac{e^{\rho} \widetilde{\gamma}}{h_{1} \cdots h_{p}}>0, \quad \text { on } \bar{M} .
\end{aligned}
$$

Indeed, (4.15) and (1.2) imply that there exists a $\widetilde{\rho} \in C^{\infty}(\bar{M})$ such that

$$
d d^{c} \log \frac{e^{\widetilde{\rho}} \widetilde{\gamma}}{h_{1} \cdots h_{p}}>0 .
$$


If $q<n$, then by (4.7), there exists a $\varphi_{I, \nu} \in C^{\infty}\left(D_{I}^{\nu}\right)$ such that

$$
\left.d d^{c} \log \frac{e^{\widetilde{\rho}} \widetilde{\gamma}}{h_{1} \cdots h_{p}}\right|_{D_{I}^{\nu}}+\left.d d^{c} \log \left(\prod_{j \notin I} \sigma_{j}^{-2}\right)\right|_{D_{I}^{\nu}}+d d^{c} \varphi_{I, \nu}=\omega_{K-E, \nu}
$$

Then, by adding a constant to $\varphi_{I, \nu}$, we get

$$
\left.\frac{e^{\tilde{\rho}} \widetilde{\gamma}}{\prod_{i=1}^{q} h_{i} \prod_{j=q+1}^{p}\left|s_{j}\right|^{2} \sigma_{j}^{2}}\right|_{D_{I}^{\nu}} \cdot e^{\varphi_{I, \nu}}=2^{q} n ! \operatorname{det}\left(g_{i \bar{j}, \nu}\right) .
$$

As for $q=n$, each $D_{I}^{\nu}$ is a point in $\bar{M}$. Let $\varphi_{I, \nu}$ be the constant such that

$$
\left.\frac{e^{\widetilde{\rho}} \widetilde{\gamma}}{\prod_{i=1}^{q} h_{i} \prod_{j=q+1}^{p}\left|s_{j}\right|^{2} \sigma_{j}^{2}}\right|_{D_{I}^{\nu}} \cdot e^{\varphi_{I, \nu}}=2^{q} n !
$$

Take $s_{i} \in S_{I}$ for each $i \in I$. Let $\chi \in C^{\infty}(\mathbb{R})$ be a cut-off function such that $\chi=1$ on $(-\infty, 1]$ and $\chi=0$ on $[2,+\infty)$. Denote by

$\varphi=\frac{1}{m} \log \left[\exp \left(m \chi\left(\sum_{i \in I} \frac{\left|s_{i}\right|^{2}}{\delta}\right)\left(\varphi_{I}+C_{I} \sum_{i \in I}\left|s_{i}\right|^{2}\right)\right)+C\left(\sum_{i \in I}\left|s_{i}\right|^{2}\right)^{3}\right]$,

where $\varphi_{I} \in C^{\infty}\left(D_{I}\right)$ is defined by $\varphi_{I}=\varphi_{I, \nu}$ on each $D_{I}^{\nu}, C_{I}>0$ is a large constant depending only on $D_{I}, \delta>0$ is a small number and $m \in \mathbb{N}$ and $C=C(\delta, m)>1$ are sufficiently large numbers. It follows from either direct calculations, or the following general theorem on metric extension, that

$$
\rho \equiv \widetilde{\rho}+\varphi
$$

satisfies both (4.16) and (4.17). Therefore, let $V=e^{\rho} \omega_{\bar{M}}^{n}$, and $\Omega$ be the volume form given by (4.1); then, $\Omega \in \mathcal{M}_{I ; S_{I}, H_{I}}$ by the above construction. Finally, let $\mathcal{M}_{I, \mathcal{H}_{I}}$ be the union of all the families $\mathcal{M}_{I ; S_{I}^{\prime}, H_{I}^{\prime}}$ which are canonically compatible with $\mathcal{M}_{I ; S_{I}, H_{I}}$.

As mentioned above, we can finish the construction of $\mathcal{M}_{I, \mathcal{H}_{I}}$ by direct calculations without using the following theorem. However, since the theorem on metric extensions may have interests of its own, we include it here.

Theorem 4.8. Let $X$ be an $n$-dimensional compact complex manifold, and $L$ an ample line bundle over $X$. Let $E \subset X$ be a subvariety of complete 
intersection, i.e.,

$$
E=\bigcap_{i=1}^{k} D_{i}, \quad 1 \leq k \leq n,
$$

where $D_{i} \subset X$ are smooth irreducible hypersurfaces that meet transversally at each point of intersection. Let $h_{E}$ be a smooth metric on $\left.L\right|_{E}$, with positive curvature form on $E$. Then, $h_{E}$ can be extended to a smooth metric $h$ on $L$, with positive curvature form on $X$.

A similar result, which works for codimension 1, i.e., $E=D_{1}$ as above, was obtained by Schumacher (see Theorem 4 in [32]). His extended metric, however, is not globally smooth, since it contains a term as $\left|s_{E}\right|^{2 / m}$, where $s_{E}$ is the holomorphic section defining the divisor $E[32$, p. 634]. This difficulity can be overcome by increasing the multiplicity of the line bundle. Furthermore, by patching a cut-off function with the global defining sections instead of local ones (see also the proof of Theorem 3.6 in Section 3.2), and applying Schumacher's lemma (see below or Lemma 3 in [32]) enables us to generalize the result to any higher codimension.

Lemma 4.9 (Schumacher). Given an open set $U$ in a complex manifold, let

$$
\mathcal{F}(f)=f d d^{c} \log f
$$

for any smooth positive function $f$ on $U$. Then, for any two such functions $f$ and $g$,

$$
\mathcal{F}(f+g) \geq \mathcal{F}(f)+\mathcal{F}(g) \quad \text { on } U
$$

Proof.

$$
\mathcal{F}(f+g)-\mathcal{F}(f)-\mathcal{F}(g)=\frac{g^{3}}{f(f+g)} d\left(\frac{f}{g}\right) \wedge d^{c}\left(\frac{f}{g}\right) \geq 0
$$

Corollary 4.10. For any finitely many smooth positive functions $f_{1}, \ldots, f_{N}$ on $U$,

$$
\mathcal{F}\left(\sum_{k=1}^{N} f_{k}\right) \geq \sum_{k=1}^{N} \mathcal{F}\left(f_{k}\right) \quad \text { on } U .
$$

Lemma 4.11. Assume that there exists an open neighborhood $U$ of $E$ and a smooth metric $h_{U}$ on $\left.L\right|_{U}$ such that $\left.h_{U}\right|_{E}=h_{E}$, and the curvature form of $h_{U}$ is positive on $U$. Then, the metric $h_{E}$ can be extended to a metric $h$ on $L$ with positive curvature form on $X$. 
Proof of Lemma 4.11. Extend $h_{U}$ (with some shrinking of $U$, if necessary) to a smooth metric $h_{1}$ of $L$ over $X$, without any curvature assumptions on $X \backslash U$. On the other hand, since $L$ is ample, there exists a metric $h_{p}$ on $L$ with positive curvature form on $X$. Let $s_{i} \in H^{0}\left(X, \mathcal{O}\left(\left[D_{i}\right]\right)\right)$ be the defining section of $D_{i}$ and locally $\left|s_{i}\right|^{2}=\left|z^{i}\right|^{2} h_{D_{i}}$ for each $i=1, \ldots, k$. Let

$$
\frac{1}{h}=\left(\frac{1}{h_{1}^{m}}+\frac{C}{h_{p}^{m}}\left(\sum_{i=1}^{k}\left|s_{i}\right|^{2}\right)^{3}\right)^{1 / m}
$$

where $m$ is a sufficiently large integer such that $h_{p}^{m} h_{D_{i}}^{-3}$ is a metric on $m L-3\left[D_{i}\right]$ with positive curvature form on $X$ for $i=1, \ldots, k$, and $C>1$ is a large constant depending only on $m$ and $U$. We claim that $h$ is the desired extension metric.

Applying Schumacher's lemma, we get

$$
\mathcal{F}\left(\frac{1}{h^{m}}\right) \geq \mathcal{F}\left(\frac{1}{h_{1}^{m}}\right)+C \cdot \mathcal{F}\left(\frac{1}{h_{p}^{m}}\left(\sum_{i=1}^{k}\left|s_{i}\right|^{2}\right)^{3}\right) \quad \text { on } X \backslash E
$$

We want to show that $\mathcal{F}\left(1 / h_{p}^{m}\left(\sum_{i=1}^{k}\left|s_{i}\right|^{2}\right)^{3}\right)$ is positive definite on $X \backslash E$. In fact, on $X \backslash D$, where $D:=D_{1}+\cdots+D_{k}$, applying Corollary 4.10 yields

$$
\begin{aligned}
\mathcal{F}\left(\frac{1}{h_{p}^{m}}\left(\sum_{i=1}^{k}\left|s_{i}\right|^{2}\right)^{3}\right) & \geq \frac{3}{h_{p}^{2 m / 3}}\left(\sum_{i=1}^{k}\left|s_{i}\right|^{2}\right)^{2} \sum_{i=1}^{k} \mathcal{F}\left(\frac{\left|s_{i}\right|^{2}}{h_{p}^{m / 3}}\right) \\
& \geq \frac{1}{h_{p}^{m}}\left(\sum_{i=1}^{k}\left|s_{i}\right|^{2}\right)^{2} \sum_{i=1}^{k}\left|s_{i}\right|^{2} d d^{c} \log \frac{h_{D_{i}}^{3}}{h_{p}^{m}}>0 .
\end{aligned}
$$

For an arbitrary point $P \in(X \backslash E) \cap D$, without loss of generality, assume that $s_{1}, \ldots, s_{l}$ pass through $P$ while $s_{l+1}, \ldots s_{k}$ do not, $1<l<k$. Note that

$$
\begin{aligned}
d d^{c} \log \left(\sum_{i=1}^{k}\left|s_{i}\right|^{2}\right) & =\frac{\sum_{i=1}^{l} h_{D_{i}} d z \wedge d \bar{z}^{i}}{\sum_{j=l+1}^{k}\left|s_{i}\right|^{2}}+d d^{c} \log \left(\sum_{j=l+1}^{k}\left|s_{i}\right|^{2}\right) \\
& >d d^{c} \log \left(\sum_{j=l+1}^{k}\left|s_{i}\right|^{2}\right) \text { at } P .
\end{aligned}
$$


Hence, applying Corollary 4.10 again, we have

$$
\mathcal{F}\left(\frac{1}{h_{p}^{m}}\left(\sum_{i=1}^{k}\left|s_{i}\right|^{2}\right)^{3}\right)>\mathcal{F}\left(\frac{1}{h_{p}^{m}}\left(\sum_{i=l+1}^{k}\left|s_{i}\right|^{2}\right)^{3}\right)>0 \text { at } P .
$$

Therefore, $\mathcal{F}\left(1 / h_{p}^{m}\left(\sum_{i=1}^{k}\left|s_{i}\right|^{2}\right)^{3}\right)$ is positive definite on $X \backslash E$.

Let $U^{\prime} \subset U$ be a relatively compact open neighborhood of $E$. On $X \backslash U^{\prime}$, for a fixed $m$, we can choose $C=C\left(U^{\prime}, m\right)>0$ sufficiently large such that the right hand side of (4.18) is positive definite, which implies that the curvature form of $h$ is positive on $X \backslash U^{\prime}$. On $U \backslash E$, by the assumption of $h_{U}$, applying (4.18) again, we know that the curvature form of $h$ is positive on $U \backslash E$. Finally, by direct calculation, we have that the curvature forms of $h$ and $h_{1}$ coincide at every point of the subvariety $E$. This completes the proof.

Proof of Theorem 4.8. By Lemma 4.11, it suffices to extend $h_{E}$ to a metric whose curvature form is positive on a neighborhood of $E$. It follows from positivity of $L$ that there exists a metric $h_{p}$ with positive curvature form $\Theta_{p}$ on $X$. Note that $\left[\left.\Theta_{p}\right|_{E}\right]=c_{1}\left(\left.L\right|_{E}\right)$. Hence, there is a $\varphi_{E} \in C^{\infty}(E)$ such that

$$
\frac{e^{\varphi_{E}}}{\left.h_{p}\right|_{E}}=\frac{1}{h_{E}}
$$

We can choose a large constant $C_{E}$ depending only on $E$ such that

$$
\Theta_{p}+d d^{c}\left(\varphi_{E}+C_{E} \sum_{i=1}^{k}\left|s_{i}\right|^{2}\right)>0
$$

at every point of $E$. Then, there exists a small constant $\delta>0$ such that (4.19) holds on $\left\{\sum_{i=1}^{k}\left|s_{i}\right|^{2}<\delta\right\}$. Let

$$
\varphi=\chi\left(\sum_{i=1}^{k} \frac{\left|s_{i}\right|^{2}}{\delta}\right)\left(\varphi_{E}+C_{E} \sum_{i=1}^{k}\left|s_{i}\right|^{2}\right)
$$

where $\chi \in C^{\infty}(\mathbb{R})$ is a non-negative cut-off function with value 1 on $(-\infty, 1)$ and value 0 on $(2,+\infty)$. Now let

$$
\frac{1}{h_{1}}=\frac{e^{\varphi}}{h_{p}}
$$


Then, $h_{1}$ is a smooth metric of $L$ whose curvature form is positive on $\left\{\sum_{i=1}^{k}\left|s_{i}\right|^{2}<\delta\right\}$. Hence, by (the proof of) Lemma 4.11, the metric

$$
\frac{1}{h}=\frac{1}{h_{p}}\left(e^{m \varphi}+C\left(\sum_{i=1}^{k}\left|s_{i}\right|^{2}\right)^{3}\right)^{1 / m}
$$

where $m \in \mathbb{N}$ and $C=C(\delta, m)>1$ are sufficiently large constants, is the desired extension metric on $X$.

\subsection{Higher canonical asymptotics of Kähler-Einstein metrics}

Finally, we will state and prove the asymptotics theorem in the case of complement of a simple normal crossing divisor.

Theorem 4.12. Suppose $I=\{1, \ldots, q\} \in \mathcal{I}$. Let $\Omega \in \mathcal{M}_{I}$. Then the solution $u$ of

$$
\begin{aligned}
& M_{\omega}(u)=J(\Omega)^{-1}, \\
& \frac{1}{C} \omega \leq \omega_{u} \leq C \omega, \quad C>0,
\end{aligned}
$$

is in $\mathcal{R}_{I, 1}^{G}(M)$. More precisely, $u \in \mathcal{R}_{I, 1}(M)$; furthermore, there is a multiple sequence $\left\{\psi_{r}\right\}_{r \in\left(\mathbb{Z}_{+}^{q} \times 0\right)} \subset \mathcal{R}(M)$ such that for any $N \in \mathbb{N}$,

$$
u-\sum_{|r|=1}^{N} \psi_{r} \sigma^{-r} \in \mathcal{R}_{I, N+1}(M) .
$$

Moreover, assume that $\Omega \in \mathcal{M}_{I, \mathcal{H}_{I}} ;$ if we start from another $\Omega^{\prime} \in \mathcal{M}_{I, \mathcal{H}_{I}}$ and get

$$
u^{\prime}-\sum_{|r|=1}^{N} \psi_{r}^{\prime} \sigma^{\prime-r} \in \mathcal{R}_{I, N+1}(M), \quad \text { for all } N \in \mathbb{N}
$$

then

$$
\begin{aligned}
u^{\prime}-u & \in C+\mathcal{R}_{I, \infty}(M) ; \\
\psi_{r}^{\prime}-\psi_{r} & \in \mathcal{R}_{I, \infty}(M), \quad \text { for all } r \in \mathbb{Z}_{+}^{q} \times 0 .
\end{aligned}
$$


Consequently, the Kähler-Einstein volume form $\Omega_{K-E}=e^{u} \Omega$ on $M$ has the following canonical asymptotic expansion near the smooth subvariety $D_{I}$ :

$$
\Omega_{K-E} \sim\left(1+\sum_{|r|=1}^{\infty} \frac{\phi_{r}}{\sigma^{r}}\right) \Omega
$$

where $\phi_{r} \in \mathcal{R}(M)$ for each $r \in\left(\mathbb{Z}_{+}^{q} \times 0\right)$. The asymptotics is canonical in the following sense: if (4.25) is derived in terms of another $\Omega^{\prime} \in \mathcal{M}_{I, \mathcal{H}_{I}}$ and coefficients $\left\{\phi_{r}^{\prime}\right\}_{r \in \mathbb{Z}_{+}^{q} \times 0}$, then

$$
\phi_{r}^{\prime}-\phi_{r} \in \mathcal{R}_{I, \infty}(M), \quad \text { for all } r \in \mathbb{Z}_{+}^{q} \times 0 .
$$

Proof of Theorem 4.12. For simplicity, we identify the index set $\mathbb{Z}_{+}^{q} \times 0$ with $\mathbb{Z}_{+}^{q}$; namely, in the following proof, $r=r_{I}=\left(r_{1}, \ldots, r_{q}\right) \in \mathbb{Z}_{+}^{q}$ and $\sigma^{-r}=\sigma^{-r_{I}}=\sigma_{1}^{-r_{1}} \cdots \sigma_{q}^{-r_{q}}$. Write

$$
\begin{aligned}
J(\Omega) & =\frac{\omega^{n}}{\Omega} \\
& =f_{0}\left(1+\sum_{|r|=1}^{n-q} \sigma^{-r} f_{r}\right)\left(1+f_{b}\right),
\end{aligned}
$$

where

$$
\begin{aligned}
& f_{0}=\frac{2^{q} n ! \omega_{K, I}^{n-q} \wedge \prod_{i=1}^{q}\left|s_{i}\right|^{2} d \sigma_{i} \wedge d^{c} \sigma_{i}}{(n-q) ! V} \prod_{j=q+1}^{p}\left|s_{i}\right|^{2} \sigma_{i}^{2} \\
& f_{b}=\frac{\sum_{j=0}^{q-1}\left(\begin{array}{c}
n \\
j
\end{array}\right)\left(\omega_{K, I}+\sum_{i=1}^{q} 2 \sigma_{i}^{-1} \omega_{c_{i}}\right)^{n-j} \wedge\left(\sum_{i=1}^{q} 2 \sigma_{i}^{-2} d \sigma_{i} \wedge d^{c} \sigma_{i}\right)^{j}}{q !\left(\begin{array}{c}
n \\
q
\end{array}\right)\left(\omega_{K, I}+\sum_{i=1}^{q} 2 \sigma_{i}^{-1} \omega_{c_{i}}\right)^{n-q} \wedge \prod_{i=1}^{q} 2 \sigma_{i}^{-2} d \sigma_{i} \wedge d^{c} \sigma_{i}} \\
& f_{r}=\frac{|r| ! 2^{|r|}\left(\begin{array}{c}
n-q \\
|r|
\end{array}\right) \omega_{K, I}^{n-q-|r|} \wedge \prod_{i=1}^{q} \omega_{c_{i}}^{r_{i}} \wedge \prod_{i=1}^{q} 2 \sigma_{i}^{-2} d \sigma_{i} \wedge d^{c} \sigma_{i}}{r !\left(\begin{array}{c}
n \\
q
\end{array}\right) \omega_{K, I}^{n-q} \wedge \prod_{i=1}^{q} 2 \sigma_{i}^{-2} d \sigma_{i} \wedge d^{c} \sigma_{i}}
\end{aligned}
$$

in which $1 \leq|r| \leq n-q$, and

$$
\omega_{K, I}=\operatorname{Ric}\left(\frac{V}{\prod_{i=1}^{p}\left|s_{i}\right|^{2} \prod_{j \notin I} \sigma_{j}^{2}}\right)=\omega_{K}-2 d d^{c} \log \left(\prod_{j=q+1}^{p} \sigma_{j}\right) .
$$

By definition, we know that $f_{r} \in C^{\infty}(\bar{M}) \subset \mathcal{R}(M), f_{b} \in \mathcal{R}_{I, \infty}(M), f_{b}>0$ on $M$, and that $f_{0} \in \mathcal{R}(M)$, is smooth near $D_{I}$, and $f_{0}>0$ on $\bar{M}$. Since 
$\Omega \in \mathcal{M}_{I}$, by (4.8) of condition (4.2), we have

$$
\left.f_{0}\right|_{D_{I}}=1 .
$$

Hence, $f_{0}-1 \in \mathcal{R}_{I, \infty}(M)$. Moreover,

$$
\sum_{|r|=k} \sigma^{-r} f_{r} \in \mathcal{R}_{I, k}(M), \quad k \geq 1 .
$$

These imply that

$$
\log J(\Omega) \in \mathcal{R}_{I, 1}(M) .
$$

Then, applying Theorem 4.1 yields that the solution

$$
u \in \mathcal{R}_{I, 1}(M) .
$$

Next, to derive (4.22), for each $r \in \mathbb{Z}_{+}^{q}$ and $|r|=1$, let $\psi_{r} \in \mathcal{R}(M)$ be the unique solution of

$$
L_{r}\left(\psi_{r}\right) \equiv \Delta_{\omega} \psi_{r}-2 \sum_{i=1}^{q} r_{i} \sigma_{i}^{-1} H\left(\sigma_{i}, \psi_{r}\right)+c_{r} \psi_{r}=-f_{r}
$$

Denote by

$$
u_{1}=\sum_{|r|=1} \psi_{r} \sigma^{-r}
$$

Then, we have

$$
\left(\Delta_{\omega}-1\right)\left(u_{1}\right)=-\sum_{|r|=1} f_{r} \sigma^{-r}
$$

Also,

$$
\begin{aligned}
J\left(e^{u_{1}} \Omega\right) & =M_{\omega}\left(u_{1}\right) J(\Omega) \\
& =\left(1+\Delta_{\omega} u_{1}+G_{2}\left(u_{1}\right)+\cdots+G_{n}\left(u_{1}\right)\right) e^{-u_{1}} J(\Omega) \\
& \in 1+\sum_{|r|=2} \mathcal{R}_{r}(M) \subset 1+\mathcal{R}_{I, 2}(M),
\end{aligned}
$$

in which

$$
G_{i}(v) \equiv \frac{\left(\begin{array}{c}
n \\
i
\end{array}\right) \omega^{n-i} \wedge\left(d d^{c} v\right)^{i}}{\omega}, \quad \text { for all } v \in \mathcal{R}(M), \quad i \geq 2
$$


By the same argument in the proof of Theorem 3.8, and applying Theorem 4.2 , we have that

$$
u-\sum_{|r|=1} \psi_{r} \sigma^{-r} \in \mathcal{R}_{I, 2}(M)
$$

Then, similar to the proof of Theorem 3.8, by induction and Theorem 4.2, we prove that there exists a sequence $\left\{\psi_{r}\right\}_{r \in \mathbb{Z}_{+}^{q}}$ such that for each $N \in \mathbb{N}$,

$$
\begin{gathered}
J\left(\exp \left(\sum_{|r|=1}^{N} \psi_{r} \sigma^{-r}\right) \cdot \Omega\right)-1 \in \sum_{|r|=N+1} \mathcal{R}_{r}(M) \subset \mathcal{R}_{I, N+1}(M), \\
u-\sum_{|r|=1}^{N} \psi_{r} \sigma^{-r} \in \mathcal{R}_{I, N+1}(M) .
\end{gathered}
$$

Therefore, this completes the proof of (4.22).

As for the canonicity, (4.23) follows from Proposition 4.4. It remains to prove (4.24). In fact, by construction

$$
f_{r}^{\prime}-f_{r} \in \mathcal{R}_{I, \infty}(M), \quad \text { for all } 1 \leq|r| \leq n-q .
$$

Furthermore, it follows from (4.11) and (4.12), in the proof of Lemma 4.7, that

$$
G_{i}^{\prime}\left(v^{\prime}\right)-G_{i}(v) \in \mathcal{R}_{I, \infty}(M), \quad \text { for all } v^{\prime}-v \in \mathcal{R}_{I, \infty}(M),
$$

where $G_{i}^{\prime}(M)$ is given by $(4.26)$ with $\omega^{\prime}$ replaced by $\omega$. Then, (4.24) follows immediately from (4.27), Proposition 4.5, (4.28) and induction.

\section{Acknowledgments}

The author would like to thank his thesis advisor, Professor Shing-Tung Yau, for introducing this problem, and providing unfailing support and encouragement, and many corrections and suggestions during the long preparation. The author would also like to thank Professor Richard Melrose for patiently explaining the ideas in [24] and helpful discussions. Moreover, the author is grateful to Xiaowei Wang, Xiaodong Wang, Chiu-Chu Melissa Liu and Bohui Chen for conversations and their moral support. Finally, the author would like to thank the referee for the careful proofreading and many suggestions to improve the presentation of this paper. The results of this paper are also contained in the author's PhD thesis at MIT. 


\section{References}

[1] T. Aubin, Équations du type Monge-Ampère sur les variétés kähleriennes compactes, C. R. Acad. Sci. Paris Sér. 283 (1976), no. 3, 119-121.

[2] S. Bando, Einstein Kähler metrics of negative Ricci curvature on open Kähler manifolds. Kähler Metrics and Moduli Spaces, Adv. Stud. in Pure Math. 18, (1990), no. 2, 105-136.

[3] M. Beals, C. Fefferman and R. Grossman, Strictly pseudoconvex domains in $\mathbb{C}^{n}$, Bull. Amer. Math. Soc. 8 (1983), 125-322.

[4] J. Bland, Local boundary behaviour of the canonical Einstein-Kähler metric on pseudoconvex domains, UCLA PhD thesis, 1982.

[5] J. Carlson and P. Griffiths, A defect relation for equidimensional holomorphic mappings between algebraic varieties, Ann. Math. 95 (1972), $557-584$.

[6] I. Chavel, Eigenvalues in Riemannian Geometry, Academic Press, New York, 1984.

[7] S.-Y. Cheng and S.-T. Yau, Differential equations on Riemannian manifolds and their geometric applications, Comm. Pure Appl. Math. 28 (1975), 333-354.

[8] S.-Y. Cheng and S.-T. Yau, On the existence of a complete Kähler metric on non-compact complex manifolds and the regularity of Fefferman's equation, Comm. Pure Appl. Math. 33 (1980), 507-544.

[9] S.-Y. Cheng and S.-T. Yau, Inequality between Chern numbers of singular Kahler surfaces and characterization of orbit space of discrete group of $S U(2,1)$, Contemp. Math. 49 (1986), 31-43.

[10] M. Cornalba and P. Griffiths, Analytic cycles and vector bundles on non-compact algebraic varieties. Invent. Math. 28 (1975), 1-106.

[11] M. Craioveanu, M. Puta and T. Rassias, Old and New Aspects in Spectral Geometry, Kluwer Academic Publishers, 2001.

[12] C. Epstein, R. Melrose and G. Mendoza, Resolvent of the Laplacian on strictly pseudoconvex domains, Acta Math. 167 (1991), 1-106.

[13] L. Evans, Parital Differential Equations, AMS Press, 1999. 
[14] C. Fefferman, The Bergman kernel and biholomorphic mappings of pseudoconvex domains, Invent. Math. 26 (1974), 1-65.

[15] C. Fefferman, Monge-Ampère equations, the Bergman kernel, and geometry of pseudoconvex domains, Ann. Math. (2) 103 (1976), 395-416.

[16] C. Fefferman, Parabolic invariant theory in complex analysis, Adv. Math. 31 (1979), 131-262.

[17] D. Gilbarg and N. Trudinger, Elliptic Partial Differential Equations of Second order, Springer, 1998.

[18] R. Graham, Higher asymptotics of the complex Monge-Ampère equation, Compo. Math. 64 (1987), 133-155.

[19] P. Griffiths and J. Harris, Principles of Algebraic Geometry, John Wiley \& Sons, 1978.

[20] P. Griffiths and J. King Nevanlinna theory and holomorphic mappings between algebraic varieties. Acta Math. 130 (1973), 145-220.

[21] K. Hirachi, Construction of boundary invariants and the logarithmic singularity of the Bergman kernel, Ann. Math. (2) 151 (2000), no. 1, 151-191.

[22] R. Kobayashi, Kähler-Einstein metric on an open algebraic manifold, Osaka J. Math. 21 (1984), 399-418.

[23] J. Lee, Higher asymptotics of the complex Monge-Ampère equation and geometry of CR manifolds, M.I.T. PhD thesis, 1982.

[24] J. Lee and R. Melrose, Boundary behaviour of the complex MongeAmpère equation, Acta Math. 148 (1982), 159-192.

[25] J. Lee and T. Parker, The Yamabe problem, Bull. Amer. Math. Soc. 17 (1987), 37-91.

[26] R. Mazzeo, Kähler-Einstein metrics singular along a smooth divisor, Journées Équations aux dérivées partielles, Saint-Jean-de-Monts, 31 mai-4 juin 1999 GDR 1151 (CNRS).

[27] R. Melrose, Differential analysis on manifolds with corners, http://www-math.mit.edu/ rbm/book.html. 
[28] Mok, N. and Yau, S. - T., Completeness of the Kähler-Einstein metric on bounded domains and the characterization of domains of holomorphy by curvature conditions, Proc. Sympos. Pure Math., 39, American Mathematical Society, Providence, RI, 1983.

[29] R. Nevanlinna, Analytic Functions, Springer-Verlag, New York, 1970.

[30] M. Protter and H. Weinberger, Maximum Principles in Differential Equations, Springer-Verlag, 1984.

[31] R. Schoen and S.-T. Yau, Lectures on Differential Geometry, International Press, 1994.

[32] G. Schumacher, Asymptotics of Kähler-Einstein metrics on quasiprojective manifolds and an extension theorem on holomorphic maps, Math. Ann. 311 (1998), no. 4, 631-645.

[33] G. Schumacher, Asymptotics of complete Kähler-Einstein metricsnegativity of the holomorphic sectional curvature, Doc. Math. 7 (2002), 653-658 (electronic).

[34] G. Tian and S.-T. Yau, Existence of Kähler-Einstein metrics on complete Kähler manifolds and their applications to algebraic geometry, Proc. Conf. San Diego. Adv. Ser. Math. Phys. 1 (1987), 574-629.

[35] H. Tsuji, An inequality of Chern numbers for open varieties, Math. Ann., 277 (1987), no. 3, 483-487.

[36] H. Wu, Old and new invariant metrics on complex manifolds, Several complex variables (Stockholm, 1987/1988), 640-682.

[37] S.-T. Yau, Harmonic functions on complete Riemannian manifolds, Comm. Pure. Appl. Math. 28 (1975), 201-228.

[38] S.-T. Yau, Calabi's conjecture and some results in algebraic geometry, Proc. Nat. Acad. Sci. USA 74 (1977), no. 5, 1798-1799.

[39] S.-T. Yau, On the Ricci curvature of a compact Kähler manifold and the complex Monge-Ampère equation I, Comm. Pure Appl. Math. 31 (1978), 339-411.

[40] S.-T. Yau, Métriques de Kähler-Einstein sur les variétés ouvertes, "Première Classe de Chern et courbure de Ricci: Preuve de la conjecture de Calabi", Séminaire Palaiseau, Astérisque, 58 (1978), 163-167. 
[41] S.-T. Yau, A splitting theorem and algebraic geometric characterization of locally Hermitian symmetric spaces. Commun. Anal. Geom. 1 (1993), no. $3,473-486$.

[42] S.-T. Yau, Review of geometry and analysis, Mathematics: Fronties and Perspectives, AMS 2000, 353-401.

Department of Mathematics

Massachusetts Institute of Technology

CAmbridge

MA 02139

USA

E-mail address: dwu@math.stanford.edu

Received August 19, 2005 
